\title{
Using Luenberger observers and dwell-time logic for feedback hybrid loops in continuous-time control systems*
}

\author{
Francesco Fichera, Christophe Prieur, Sophie Tarbouriech and Luca Zaccarian ${ }^{\dagger}$
}

\begin{abstract}
For linear control systems, we add a Luenberger observer to extend the state feedback hybrid loop designs of (Prieur et al., NOLCOS 2010) and (Fichera et al., ADHS 2012), to the output feedback case. This defines an output feedback controller. The hybrid loop consists in a continuous-time dynamics when the estimate of the state leads to a non-increase of a suitable Lyapunov-like function, and in a jump equation when this condition is not satisfied. The jump equation is defined by means of a static state feedback law from the observed state. Four techniques based on different reset maps and flow and jump sets are proposed, all of them exploiting a suitable dwell-time logic. For the proposed designs we prove global exponential stability of the origin. The effectiveness of the proposed solutions is illustrated by simulation examples where we show suitable reduction of the output overshoot.
\end{abstract}

Index Terms - hybrid control, hybrid Lyapunov function, reset control

\section{Introduction}

In the quest of providing more flexible stabilizing controllers and more efficient design techniques, research efforts have focused on developing control algorithms using controllers that involve switching or on-line adaptation, leading to dynamic controllers whose states can experience jumps (namely, hybrid controllers). Such a flexibility may overcome some fundamental limitations of linear control (see [4] for an example, and see $[18,19,24,30]$ for nonlinear controls) and improve the performance of linear closed-loop systems (see [9-11,26]). A particular class of hybrid controllers of interest for this paper is the so-called reset controllers class where a part of the state is reset when it satisfies some algebraic conditions. See $[3,6,9,23]$ where reset controllers are shown to decrease the $\mathcal{L}_{2}$ gain between perturbations and the output. Consider also [1] where it is shown that reset controllers may be useful to improve the $\mathcal{L}_{2}$ or $\mathcal{H}_{2}$ stability of linear systems.

Generally, a hybrid controller follows a continuous-time dynamical equation during flow, and a difference equation which governs the way the states are instantaneously changed during jumps. As first proposed in [25], the flow and

\footnotetext{
*Work supported by HYCON2 Network of Excellence "Highly-Complex and Networked Control Systems", grant agreement 257462.

${ }^{\dagger}$ F. Fichera, S. Tarbouriech and L. Zaccarian are with CNRS, LAAS, 7 avenue du colonel Roche, F-31400 Toulouse, France and Univ. de Toulouse, LAAS, F-31400 Toulouse, France (email: \{ffichera,tarbour,lzaccari\}@laas.fr)

C. Prieur is with Department of Automatic Control, Gipsa-lab, 961 rue de la Houille Blanche, BP 46, 38402 Grenoble Cedex, France (email: christophe.prieur@gipsa-lab.grenoble-inp.fr)
} 
jump region may be governed by suitable Lyapunov-like conditions, and the state of the controller may be reset to a value given by a pre-defined static state feedback controller. These techniques are also used in the event-triggered control literature (see $[7,8,20]$ and see $[2,29]$ in a nonlinear context).

The results of [25-27] propose hybrid loops that augment linear continuous-time dynamic controllers for a continuoustime plant with the property of guaranteeing the non-increase of suitable Lyapunov-like functions, together with asymptotic stability of the origin. In particular, these hybrid loops can be understood as peculiar reset rules for the controller state which are triggered whenever the closed-loop state enters a specific region. The Lyapunov-like functions, considered in [25-27] for linear control systems, are quadratic and the jump and flow sets are symmetric cones. Moreover, it is shown in [26] that this architecture allows, for example, to reduce the output overshoot in linear control systems. A drawback of the approach in [25-27] is that the hybrid loops (namely the reset rules) require knowledge of the full state of the closed loop, to establish whether it belongs to the jump or to the flow set. To overcome this drawback, a natural approach is to use an observer, to estimate the plant state, and to use this estimate when closing the hybrid loop. Such a separation principle only holds locally for nonlinear hybrid control systems see [31], however we succeed to propose in this paper a scheme consisting in a hybrid output feedback controller embedding a Luenberger observer which recovers asymptotically and globally the original scheme of [25-27] as the observation error converges to zero. The introduction of an observer in the scheme generates a number of problems arising from the fact that the Zeno solutions occurring at the origin of the scheme of [25-27] become non-converging Zeno solutions in the presence of the observer. To avoid this defective phenomenon, paralleling the results in [12], we recently proposed in [10] a control scheme where an arbitrarily small ball centered in the origin is removed from the jump set, so that, close to the origin, the observer is allowed to flow and to drive to zero the observation error. As a consequence, the asymptotic stability results of [25-27] become practical asymptotic stability results as it is proved that the closed loop converges to this ball. Moreover, since convergence to zero of the plant state is not guaranteed anymore, the results of [26, Theorem 2] could not be recovered in [10], thus providing a partial result for the output feedback case.

In this paper we address the output feedback extension of [25-27] using a different approach from [10]. Indeed we remove the defective Zeno solutions by augmenting the control system with a dwell-time logic which enforces a suitable dwell time between each pair of consecutive jumps (or resets). The approach is inspired by the proof technique in [15, Example 27] and parallels the results of [13]. We extend the results of [11] where a dwell-time logic was introduced in the schemes of [25-27] to recover global exponential stability of the origin by using the results in [32]. As in [11], we show here that when introducing the dwell-time logic, as long as the dwell-time parameter is sufficiently small, we recover asymptotically (as the observation error converges to zero) all the solutions generated by the hybrid closed loops of [25-27]. In other words, the dwell-time logic has the useful property of removing the defective Zeno solutions from the set of solutions of the control scheme while not removing any other solution from the solution set of the original system.

The paper is structured as follows. In Section 2 the control architecture under consideration and the main results are stated. A practical example is presented in Section 3 and some simulations are performed by applying some of the results proven in this paper, and by using an exact observer and a reduced order observer based on a reduced order approximation of the plant dynamics. Section 4 collects the proofs of the results. A conclusion and some open questions are presented in Section 5. 
Notation and preliminaries. Given a vector $x, x^{T}$ denotes the transpose of $x$. The Euclidean norm of a vector is denoted by $|\cdot|$ and the scalar product is denoted by $\langle\cdot, \cdot\rangle$. $\mathbb{R}$ denotes the set of real numbers, $\mathbb{Z}$ denotes the set of integers. Moreover, $\mathbb{R}_{\geq 0}$ (resp. $\mathbb{Z}_{\geq 0}$ ) denotes the set of non-negative real numbers (resp. the set of nonnegative integers). For a matrix $M, \operatorname{He}(M)=M+M^{T}$. For a positive integer $n, I_{n}$ (respectively, $0_{n}$ ) denotes the identity matrix (respectively, the null matrix) in $\mathbb{R}^{n \times n} .0_{n, m}$ with $n \neq m$ and $n$, $m$ positive integers, denotes the null matrix in $\mathbb{R}^{n \times m}$. The subscripts may be omitted when there is no ambiguity. Given a compact set $\mathcal{A}$, the notation $|x|_{\mathcal{A}}=\min \{|x-y|: y \in \mathcal{A}\}$ indicates the distance of the vector $x$ from the set $\mathcal{A}$. If $\mathcal{A}$ is the origin then $|x|_{\mathcal{A}}=|x|$. For any $s \in \mathbb{R}$, the function $\mathrm{dz}: \mathbb{R} \rightarrow \mathbb{R}$ is defined by $\mathrm{dz}(s)=0$ if $|s| \leq 1$ and $\operatorname{dz}(s)=\operatorname{sgn}(s)(|s|-1)$ if $|s| \geq 1$. For an introduction of the framework of hybrid systems that is considered in this paper, see, e.g., the recent works [15, 16] or the brief overview in $[21, \S \mathrm{II}]$. As specified in [32], a closed set $\mathcal{A} \subset \mathbb{R}^{n}$ is globally exponentially stable (GES) if there exist positive numbers $k, \lambda$, such that each solution satisfies

$$
|x(t, j)|_{\mathcal{A}} \leq k \exp (-\lambda(t+j))|x(0,0)|_{\mathcal{A}}, \quad \forall(t, j) \in \operatorname{dom}(x),
$$

where $|x|_{\mathcal{A}}$ is the distance of $x \in \mathbb{R}^{n}$ from the set $\mathcal{A}$ and $\operatorname{dom}(x)$ denotes the hybrid time domain of the solution $x$.

\section{Controllers architecture and statement of the main results}

\section{$2.1 \quad$ Controller architecture}

According to the problem statement in [25-27] restricted to the linear case, we consider a linear time-invariant plant $\mathcal{P}$, represented by

$$
\mathcal{P}\left\{\begin{array}{l}
\dot{x}_{p}=\bar{A}_{p} x_{p}+\bar{B}_{p} u \\
y_{p}=\bar{C}_{p} x_{p}+\bar{D}_{p} u
\end{array}\right.
$$

with $x_{p} \in \mathbb{R}^{n_{p}}, u \in \mathbb{R}^{p}$ and $y_{p} \in \mathbb{R}^{q}$.

For the plant (1) we use the controller architecture shown in Figure 1, where the dynamic controller originally considered in [25-27], whose state is denoted by $x_{c}$, is augmented with a Luenberger observer, whose state is denoted

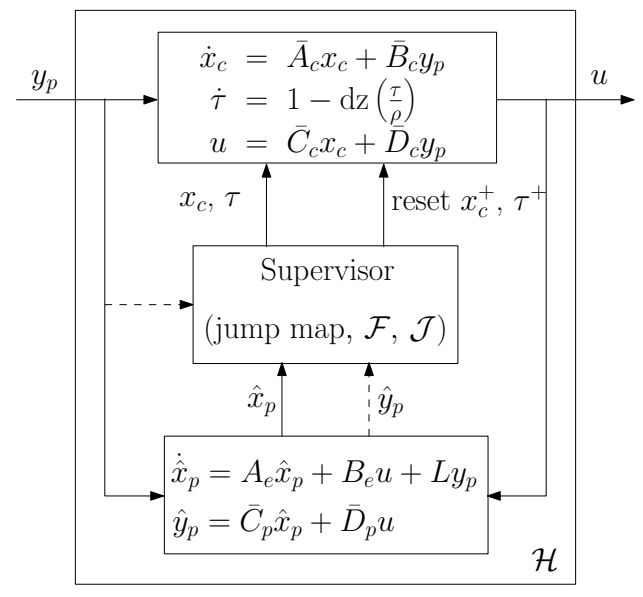

Figure 1: The proposed control scheme. 
by $\hat{x}_{p}$, with a dwell-time logic whose state is denoted by $\tau$ and with a supervisor enforcing the reset rules induced by the hybrid loops. In particular, while the linear closed loop between the dynamic controller and plant (1) does not make any use of the observed state, the observer state is used by the supervisor to decide when and where to reset the controller state.

In the next sections we will propose two solutions for the output feedback extension of the two architectures of [25-27] (thereby resulting in four schemes). All of the proposed schemes share the same flow dynamics which is

$$
\begin{aligned}
{\left[\begin{array}{c}
\dot{\hat{x}}_{p} \\
\dot{x}_{c}
\end{array}\right] } & =\left[\begin{array}{cc}
A_{e} & B_{e} \bar{C}_{c} \\
0 & \bar{A}_{c}
\end{array}\right]\left[\begin{array}{c}
\hat{x}_{p} \\
x_{c}
\end{array}\right]+\left[\begin{array}{c}
B_{e} \bar{D}_{c}+L \\
\bar{B}_{c}
\end{array}\right] y_{p} \\
& :=A_{\mathcal{H}}\left[\begin{array}{c}
\hat{x}_{p} \\
x_{c}
\end{array}\right]+B_{\mathcal{H}} y_{p} \\
u & =\left[\begin{array}{ll}
0 & \bar{C}_{c}
\end{array}\right]\left[\begin{array}{c}
\hat{x}_{p} \\
x_{c}
\end{array}\right]+\bar{D}_{c} y_{p} \\
& :=C_{\mathcal{H}}\left[\begin{array}{c}
\hat{x}_{p} \\
x_{c}
\end{array}\right]+D_{\mathcal{H}} y_{p},
\end{aligned}
$$

where $A_{e}=\bar{A}_{p}-L \bar{C}_{p}, B_{e}=\bar{B}_{p}-L \bar{D}_{p}$ and all the other matrices are design parameters that will be defined later. We assume that the Luenberger observer gain $L$ and the feedback interconnection of the plant-controller pair satisfy the following assumption.

Assumption 1 The interconnection (1)-(2) is well-posed, that is, the matrix $\left(I-\bar{D}_{p} \bar{D}_{c}\right)$ is non-singular. Moreover, the observer gain $L$ is such that the matrix $A_{e}=\bar{A}_{p}-L \bar{C}_{p}$ is Hurwitz.

Remark 1 Note that Assumption 1 implies that the pair $\left(\bar{C}_{p}, \bar{A}_{p}\right)$ is detectable.

To enforce a desirable dwell time between each pair of consecutive jumps of the hybrid control systems proposed next, we augment the state of the controller (2a), (2b) with a timer (or dwell-time logic) $\tau \in \mathbb{R}$ (see also [5, Proposition 1.1]). For the results in [32] to be applicable, it will be important that this timer lies in a compact set. Therefore, instead of simply using $\dot{\tau}=1$ for our timer, we impose the following flow dynamics, similar to [13]:

$$
\dot{\tau}=1-\mathrm{dz}\left(\frac{\tau}{\rho}\right)
$$

where $\rho>0$ is the dwell time and $\operatorname{dz}(\cdot)$ is the standard unit deadzone. From (2c), it easy to see that the right-hand side is Lipschitz, that $\dot{\tau}=1$ if $\tau \in[0, \rho]$ and that the nonnegative interval $[0,2 \rho]$ is forward invariant. The timer $\tau$ will be restricted to the set $[0,2 \rho]$, by introducing reset to zero at each jump and by terminating any solution with $\tau \notin[0,2 \rho]$. Moreover, the timer will be used to inhibit jumps whenever $\tau \in[0, \rho)$.

For later use, after defining the observation error $e:=x_{p}-\hat{x}_{p}$ and the aggregated state $x:=\left[\hat{x}_{p}^{T} x_{c}^{T} e^{T}\right]^{T} \in \mathbb{R}^{n}$, with $n=2 n_{p}+n_{c}$, it is useful to rewrite the interconnection (1), (2) in the following compact form

$$
\left[\begin{array}{c}
\dot{\hat{x}}_{p} \\
\dot{x}_{c} \\
\dot{e}
\end{array}\right]=\left[\begin{array}{ccc}
A_{p} & B_{p} & B_{o} \\
B_{c} & A_{c} & B_{c} \\
0 & 0 & A_{e}
\end{array}\right]\left[\begin{array}{c}
\hat{x}_{p} \\
x_{c} \\
e
\end{array}\right]:=A x
$$




$$
\begin{aligned}
\dot{\tau} & =1-\mathrm{dz}\left(\frac{\tau}{\rho}\right) \\
y_{p} & =\left[\begin{array}{lll}
C_{p} & C_{c} & C_{p}
\end{array}\right] x:=C x,
\end{aligned}
$$

with

$$
\left[\begin{array}{c|c|c}
A_{p} & B_{p} & B_{o} \\
\hline B_{c} & A_{c} & B_{c} \\
\hline C_{p} & C_{c} & C_{p}
\end{array}\right]=\left[\begin{array}{c|c|c}
\bar{A}_{p} & \bar{B}_{p} \bar{C}_{c} & L \bar{C}_{p} \\
\hline 0 & \bar{A}_{c} & 0 \\
\hline 0 & 0 & 0
\end{array}\right]+\left[\begin{array}{c}
\bar{B}_{p} \bar{D}_{c} \\
\hline \bar{B}_{c} \\
\hline I
\end{array}\right] X\left[\bar{C}_{p}\left|\bar{D}_{p} \bar{C}_{c}\right| \bar{C}_{p}\right],
$$

where $X=\left(I-\bar{D}_{p} \bar{D}_{c}\right)^{-1}$ is well defined and $A_{e}$ is Hurwitz from Assumption 1.

Remark 2 From the cascaded structure of the closed-loop (3a) and the fact that $A_{e}$ is Hurwitz, it is evident that $\dot{e}=\dot{x}_{p}-\dot{\hat{x}}_{p}=A_{e} e$, namely the observer state $\hat{x}_{p}$ converges exponentially to the plant state $x_{p}$, during flow.

\subsection{Reset rule replacing $x_{p}$ by $\hat{x}_{p}$}

The first state feedback reset rule proposed in [25, Theorem 1] (see also [25, Proposition 1] for the linear case addressed here) hinges upon the availability of a Lyapunov-like function $V\left(x_{p}, x_{c}\right)$ which admits a sufficiently smooth minimizer $\phi\left(x_{p}\right)=\operatorname{argmin}_{x_{c}} V\left(x_{p}, x_{c}\right)$ satisfying

$$
V\left(x_{p}, \phi\left(x_{p}\right)\right) \leq V\left(x_{p}, x_{c}\right), \quad \forall x_{c} \in \mathbb{R}^{n_{c}} .
$$

In particular, when focusing on the linear case and using quadratic Lyapunov functions $V\left(x_{p}, x_{c}\right):=\left[\begin{array}{l}x_{p} \\ x_{c}\end{array}\right]^{T} P\left[\begin{array}{l}x_{p} \\ x_{c}\end{array}\right]:=$ $\left[\begin{array}{l}x_{p} \\ x_{c}\end{array}\right]^{T}\left[\begin{array}{cc}P_{p} & P_{p c} \\ P_{p c}^{T} & P_{c}\end{array}\right]\left[\begin{array}{c}x_{p} \\ x_{c}\end{array}\right]$, with $P=P^{T}>0$, since the minimizer can be explicitly computed as $\phi\left(x_{p}\right):=-P_{c}^{-1} P_{p c}^{T} x_{p}$, property (4) reduces to (see also [25, eq. (18)])

$$
\operatorname{He}\left(\bar{P}_{p}\left(A_{p}+B_{p} K_{p}\right)\right)<-\alpha \bar{P}_{p}, \quad \bar{P}_{p}=\bar{P}_{p}^{T}>0,
$$

where

$$
\bar{P}_{p}:=P_{p}-P_{p c} P_{c}^{-1} P_{p c}^{T}, \quad K_{p}:=-P_{c}^{-1} P_{p c}^{T}
$$

and $\alpha \geq 0$ is a scalar design parameter. Note that by standard congruence transformations, constraint (5) on the entries of $P$ can be convexified (see $[26, \S 4.1]$ ).

To extend the corresponding construction to the output feedback case, we will make the same assumption here.

Assumption 2 The plant $\mathcal{P}$ in (1), and the matrix $P$ are such that equation (5), with the definitions in (6), is satisfied for some $\alpha>0$.

Using the definitions in (2), we select the hybrid controller of Figure 1 as

$$
\left\{\begin{aligned}
{\left[\begin{array}{c}
\dot{x}_{p} \\
\dot{x}_{c}
\end{array}\right] } & =A_{\mathcal{H}}\left[\begin{array}{c}
\hat{x}_{p} \\
x_{c}
\end{array}\right]+B_{\mathcal{H}} y_{p} \quad \text { if }\left(\hat{x}_{p}, x_{c}\right) \in \mathcal{F} \text { or } \tau \in[0, \rho] \\
\dot{\tau} & =1-\mathrm{dz}\left(\frac{\tau}{\rho}\right)
\end{aligned}\right.
$$




$$
\begin{aligned}
& \left\{\begin{aligned}
{\left[\begin{array}{c}
\hat{x}_{p}^{+} \\
x_{c}^{+}
\end{array}\right] } & =\left[\begin{array}{cc}
I & 0 \\
K_{p} & 0
\end{array}\right]\left[\begin{array}{c}
\hat{x}_{p} \\
x_{c}
\end{array}\right] \quad \text { if }\left(\hat{x}_{p}, x_{c}\right) \in \mathcal{J} \text { and } \tau \in[\rho, 2 \rho] \\
\tau^{+} & =0
\end{aligned}\right. \\
& u=C_{\mathcal{H}}\left[\begin{array}{c}
\hat{x}_{p} \\
x_{c}
\end{array}\right]+D_{\mathcal{H}} y_{p}
\end{aligned}
$$

with the flow and jump sets chosen as:

$$
\begin{aligned}
& \mathcal{F}=\left\{\left[\begin{array}{l}
\hat{x}_{p} \\
x_{c}
\end{array}\right]:\left[\begin{array}{l}
\hat{x}_{p} \\
x_{c}
\end{array}\right]^{T} N\left[\begin{array}{l}
\hat{x}_{p} \\
x_{c}
\end{array}\right] \leq-\tilde{\alpha}\left[\begin{array}{c}
\hat{x}_{p} \\
x_{c}
\end{array}\right]^{T} P\left[\begin{array}{l}
\hat{x}_{p} \\
x_{c}
\end{array}\right]\right\}, \\
& \mathcal{J}=\left\{\left[\begin{array}{l}
\hat{x}_{p} \\
x_{c}
\end{array}\right]:\left[\begin{array}{c}
\hat{x}_{p} \\
x_{c}
\end{array}\right]^{T} N\left[\begin{array}{c}
\hat{x}_{p} \\
x_{c}
\end{array}\right] \geq-\tilde{\alpha}\left[\begin{array}{c}
\hat{x}_{p} \\
x_{c}
\end{array}\right]^{T} P\left[\begin{array}{l}
\hat{x}_{p} \\
x_{c}
\end{array}\right]\right\},
\end{aligned}
$$

where $0<\tilde{\alpha} \leq \alpha$ and

$$
N:=\mathrm{He}\left(P\left[\begin{array}{ll}
A_{p} & B_{p} \\
B_{c} & A_{c}
\end{array}\right]\right) .
$$

Using the compact representation (3), the interconnection (1), (7) becomes the following hybrid closed-loop system:

$$
\begin{aligned}
& \begin{cases}\dot{x}=A x & \text { if } x \in\left(\mathcal{F} \times \mathbb{R}^{n_{p}}\right) \text { or } \tau \in[0, \rho] \\
\dot{\tau}=1-\mathrm{dz}\left(\frac{\tau}{\rho}\right) & \\
x^{+}=G x & \text { if } x \in\left(\mathcal{J} \times \mathbb{R}^{n_{p}}\right) \text { and } \tau \in[\rho, 2 \rho] \\
\tau^{+}=0 & \end{cases} \\
& y_{p}=C x \text {, }
\end{aligned}
$$

where

$$
G:=\left[\begin{array}{ccc}
I & 0 & 0 \\
K_{p} & 0 & 0 \\
0 & 0 & I
\end{array}\right] .
$$

The closed-loop system (8), (9) generalizes to the output feedback case the state-feedback solution whose properties are established in [25, Proposition 1]. The following theorem establishes the global exponential stability of the scheme.

Theorem 1 Consider a plant-controller pair (1), (7) satisfying Assumption 1 and two parameters $P$, $\alpha$ satisfying Assumption 2. Then there exists $\rho^{*}>0$ such that for all $\rho \in\left(0, \rho^{*}\right]$, the set

$$
\mathcal{A}:=\{0\} \times[0,2 \rho] \subset \mathbb{R}^{n} \times \mathbb{R}
$$

is globally exponentially stable for the closed-loop (9) with the sets $\mathcal{F}$ and $\mathcal{J}$ in (8).

We focus now on the second state feedback reset rule proposed in [25, Theorem 1] (see also [25, Proposition 2] and $[26,27]$ for the linear case addressed here). For this second case, the dynamics of the controller (9) unchanged but the jump and flow sets are defined based on a state feedback gain $K_{p} \in \mathbb{R}^{p \times n_{p}}$ for the plant (1) and a Lyapunov-like function which is only nonzero in the direction of the plant states, namely $V_{p}\left(x_{p}\right):=x_{p}^{T} \bar{P}_{p} x_{p}$, where $\bar{P}_{p}$ is symmetric and positive definite. Also for this second case we make the following assumption, corresponding to [25, Assumption 2] for the linear case (see also [26, eq. (7)]). 
Assumption 3 The plant $\mathcal{P}$ in (1) and the matrix pair $\left(\bar{P}_{p}, K_{p}\right)$ are such that equation (5) is satisfied for some $\alpha>0$.

Based on the matrix pair $\left(\bar{P}_{p}, K_{p}\right)$ satisfying Assumption 3 and a (typically small) scalar $\epsilon>0$, we can now define the jump and flow sets associated with the second solution and to be used in the dynamics (9):

$$
\begin{aligned}
& \mathcal{F}=\left\{\left[\begin{array}{l}
\hat{x}_{p} \\
x_{c}
\end{array}\right]:\left[\begin{array}{l}
\hat{x}_{p} \\
x_{c}
\end{array}\right]^{T} N_{p}\left[\begin{array}{c}
\hat{x}_{p} \\
x_{c}
\end{array}\right] \leq-\tilde{\alpha} \hat{x}_{p}^{T} \bar{P}_{p} \hat{x}_{p}-\epsilon\left|x_{c}\right|^{2}\right\}, \\
& \mathcal{J}=\left\{\left[\begin{array}{l}
\hat{x}_{p} \\
x_{c}
\end{array}\right]:\left[\begin{array}{c}
\hat{x}_{p} \\
x_{c}
\end{array}\right]^{T} N_{p}\left[\begin{array}{c}
\hat{x}_{p} \\
x_{c}
\end{array}\right] \geq-\tilde{\alpha} \hat{x}_{p}^{T} \bar{P}_{p} \hat{x}_{p}-\epsilon\left|x_{c}\right|^{2}\right\},
\end{aligned}
$$

where $0<\tilde{\alpha} \leq \alpha$ and

$$
N_{p}:=\operatorname{He}\left(\left[\begin{array}{cc}
\bar{P}_{p} A_{p} & \bar{P}_{p} B_{p} \\
0 & 0
\end{array}\right]\right) .
$$

The closed-loop system (9), (11) generalizes to the output feedback case the state-feedback solution whose properties are established in [26, Theorem 1] (see also [25, Proposition 2]). However, an extra term is added to allow inserting the dwell-time logic in the scheme without compromising the stability properties established in [25-27]. Indeed, while the flow and jump sets in (8) coincide with those of [25, Proposition 1], it is not true that the flow and jump sets in (11) coincide with those of [25, Proposition 2] and [26]. The difference stands in the term $-\epsilon\left|x_{c}\right|^{2}$ introduced here to provide a sufficient level of robustness. Such a robustness is required to tolerate the inevitable perturbations introduced by the dwell-time logic, which forces the system to flow even though $x$ belongs to $\mathcal{J}$ when the timer $\tau$ is too small. Note that the addition of this term does not restrict the class of systems that we consider, while it may lead to slightly smaller flow sets.

Although we do not have a formal proof of fragility of the scheme in [25, Proposition 2], we should emphasize that the proofs of stability in [25, Proposition 2] were based on the invariance principle because Lyapunov arguments only allowed to establish negative semidefiniteness of our candidate Lyapunov functions. It turns out that the term $-\epsilon\left|x_{c}\right|^{2}$ provides the missing decrease and significantly simplifies the proof of exponential stability of the state-feedback case here presented. Moreover the strict decrease arising from this term allows us to introduce the dwell-time logic without destroying the exponential stability of the closed loop. It should also be emphasized that the dwell-time parameter $\rho^{*}$ established in the next theorem shrinks to zero as $\epsilon$ becomes smaller. Perhaps, this suggests that the amount of the dwell-time perturbation that the hybrid closed loop can tolerate becomes smaller as $\epsilon$ in (11) shrinks to zero. To illustrate the effect of the new term $-\epsilon\left|x_{c}\right|^{2}$ in (11), assume without loss of generality that $e=0$ (that is $x_{p}=\hat{x}_{p}$ ) and consider the following example

$$
A=\left[\begin{array}{ll}
A_{p} & B_{p} \\
B_{c} & A_{c}
\end{array}\right]=\left[\begin{array}{cc}
-1 & 0 \\
1 & 0
\end{array}\right], \quad \bar{P}_{p}=1,
$$

which, regardless of $K_{p}$ satisfies (5) for any $\alpha<1$ (because $B_{p}=0$ ). This example does not satisfy the detectability condition in [26, Theorem 1] and indeed one can see that setting $\epsilon=0$ in (11) (thus recovering the scheme of [26]) the system starting from $\left(x_{p}, x_{c}\right)=(0, a)$ for any $a \neq 0$ can flow indefinitely so that $x_{c}(t, j)=a \neq 0$ for all times, implying no convergence (even with dwell time). 
Consider now the sets (11) with $\epsilon \neq 0$ and $\left(x_{p}, x_{c}\right)=(0, a) \notin \mathcal{F}$ because $-\epsilon\left|x_{c}\right|^{2}=-\epsilon|a|^{2}<0$. Then $x_{c}$ is instantaneously forced to jump to $x_{c}^{+}=K_{p} x_{p}=0$, regardless of $K_{p}$, and this shows convergence. In other words, the extra term $-\epsilon\left|x_{c}\right|^{2}$ appearing in (11) (combined with the dwell-time logic) ensures that, upon convergence to zero of $x_{p}$, if $x_{c} \neq 0$, then the controller will eventually be forced to jump (as $x_{p}$ gets small enough) and the $x_{c}$ substate will be stabilized by way of the jumps without the need of the detectability of $\left(B_{p}, A_{c}\right)$ required in [26, Theorem 1].

This last result is formalized in the next theorem which establishes global exponential stability of the scheme.

Theorem 2 Consider a plant-controller pair (1), (7) satisfying Assumption 1 and three parameters $\bar{P}_{p}, K_{p}$ and $\alpha$ satisfying Assumption 3. Then, for any $\epsilon>0$, there exists $\rho^{*}>0$ such that for all $\rho \in\left(0, \rho^{*}\right]$, the set $\mathcal{A}$ in $(10)$ is globally exponentially stable for the closed-loop (9) with the sets $\mathcal{F}$ and $\mathcal{J}$ in (11).

\subsection{Enhanced reset rule exploiting $y_{p}-\hat{y}_{p}$}

The solution presented in the previous section succeeds in extending the schemes of [25-27] to the output feedback case, but it does not directly exploit the instantaneous knowledge of the output error $y_{p}-\hat{y}_{p}$ for the selection of the flow and jump sets and the reset rule. We explore this additional potential here and propose an enhanced scheme which is expected to behave better during the observer transient. The two enhanced schemes rely once again on the parameters $P, \alpha$ (respectively, the matrices $\bar{P}_{p}, K_{p}, \alpha$ ) satisfying Assumption 2 (respectively, Assumption 3), plus a set of four extra matrices: $K_{y} \in \mathbb{R}^{n_{c} \times q}$ used in the jump rule and $K_{x} \in \mathbb{R}^{n_{p} \times q}, K_{c} \in \mathbb{R}^{n_{c} \times q}, K_{\eta} \in \mathbb{R}^{q \times q}$ used in the flow and jump sets definition. The four matrices represent a set of additional free tuning parameters.

Let us define $\eta:=y_{p}-\hat{y}_{p}=\bar{C}_{p}\left(x_{p}-\hat{x}_{p}\right)=\bar{C}_{p} e$ and $\zeta:=\left[\hat{x}_{p}^{T} x_{c}^{T} \eta^{T}\right]^{T}$, the hybrid controller of Figure 1 is selected as:

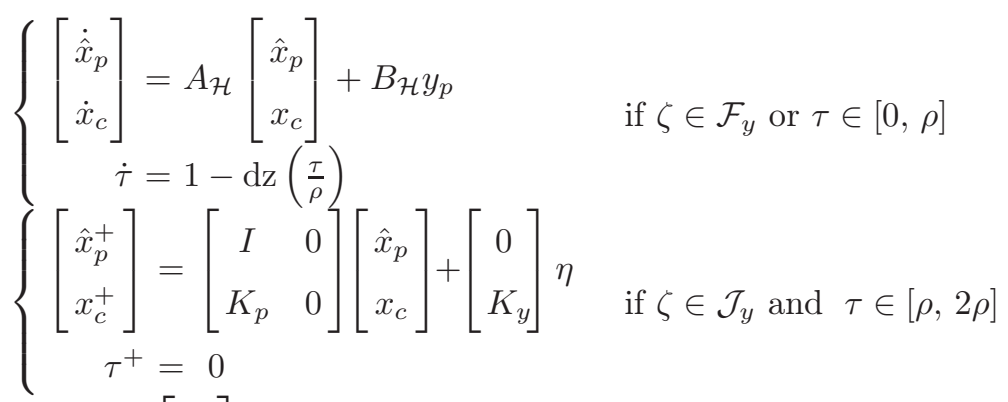

$$
\begin{aligned}
& u=C_{\mathcal{H}}\left[\begin{array}{l}
\hat{x}_{p} \\
x_{c}
\end{array}\right]+D_{\mathcal{H}} y_{p}
\end{aligned}
$$

with the flow and jump sets selected as

$$
\begin{aligned}
& \mathcal{F}_{y}=\left\{\zeta:\left[\begin{array}{c}
\hat{x}_{p} \\
x_{c}
\end{array}\right]^{T}(N+\tilde{\alpha} P)\left[\begin{array}{c}
\hat{x}_{p} \\
x_{c}
\end{array}\right] \leq\left[\begin{array}{c}
\hat{x}_{p} \\
x_{c} \\
\eta
\end{array}\right]^{T}\left[\begin{array}{c}
K_{x} \\
K_{c} \\
K_{\eta}
\end{array}\right] \eta\right\} \\
& \mathcal{J}_{y}=\left\{\zeta:\left[\begin{array}{c}
\hat{x}_{p} \\
x_{c}
\end{array}\right]^{T}(N+\tilde{\alpha} P)\left[\begin{array}{c}
\hat{x}_{p} \\
x_{c}
\end{array}\right] \geq\left[\begin{array}{c}
\hat{x}_{p} \\
x_{c} \\
\eta
\end{array}\right]^{T}\left[\begin{array}{c}
K_{x} \\
K_{c} \\
K_{\eta}
\end{array}\right] \eta\right\}
\end{aligned}
$$

where $0<\tilde{\alpha} \leq \alpha$ and $N$ and $P$ are selected according to the previous section. 
Using (3), the interconnection (1), (12) becomes the following hybrid closed-loop system:

$$
\begin{aligned}
& \begin{array}{l} 
\begin{cases}\dot{x}=A x & \text { if } \zeta \in \mathcal{F}_{y} \text { or } \tau \in[0, \rho] \\
\dot{\tau}=1-\mathrm{dz}\left(\frac{\tau}{\rho}\right) & \end{cases} \\
\begin{cases}x^{+}=G_{y} x & \text { if } \zeta \in \mathcal{J}_{y} \text { and } \tau \in[\rho, 2 \rho] \\
\tau^{+}=0 & \end{cases}
\end{array} \\
& y_{p}=C x
\end{aligned}
$$

where

$$
G_{y}:=\left[\begin{array}{ccc}
I & 0 & 0 \\
K_{p} & 0 & K_{y} \bar{C}_{p} \\
0 & 0 & I
\end{array}\right] .
$$

Once again, the closed-loop system (13), (14) generalizes to the output feedback case the state-feedback solution whose properties are established in [25, Proposition 1]. The following theorem establishes the global exponential stability of the scheme.

Theorem 3 Consider a plant-controller pair (1), (12) satisfying Assumption 1, two parameters $P$, $\alpha$ satisfying Assumption 2 and four parameters $K_{y}, K_{x}, K_{c}, K_{\eta}$. Then there exists $\rho^{*}>0$ such that for all $\rho \in\left(0, \rho^{*}\right]$, the set $\mathcal{A}$ in (10) is globally exponentially stable for the closed-loop (14) with the sets $\mathcal{F}_{y}$ and $\mathcal{J}_{y}$ in (13).

It should be emphasized that Theorem 3 only establishes the stability properties of the output feedback solution proposed here, whereas it does not highlight its strong relation with the parallel state-feedback solution of [25-27]. Such a relation is established in the next proposition. As in [17], we say that two solutions are $(T, J, \varepsilon)$-close if their graphs are $\varepsilon$-close in the compact hybrid time domain bounded by $(T, J)$ (see [17] for details). Moreover, we say that two solutions are $\varepsilon$-close if they are $(T, J, \varepsilon)$-close for all $(T, J) \in \mathbb{R}_{\geq 0} \times \mathbb{Z}_{\geq 0}$.

Proposition 1 Consider the state feedback hybrid closed loop in [25, Proposition 1]. There exists $\rho^{*}>0$ such that for all $\rho \in\left(0, \rho^{*}\right]$ the following hold:

1. any solution of the state feedback hybrid closed loop starting from $\left(x_{p}(0,0), x_{c}(0,0)\right)=\left(x_{p 0}, x_{c 0}\right)$, with $x_{p 0} \neq 0$, is also the $\left(x_{p}, x_{c}\right)$-component of a solution of the output feedback hybrid closed-loop system (13), (14) (resp. (8), (9)) starting from $\xi(0,0)=\left(\hat{x}_{p}(0,0), x_{c}(0,0), e(0,0), \tau(0,0)\right)=\left(x_{p 0}, x_{c 0}, 0, \tau_{0}\right)$ with $\tau_{0} \geq \rho$;

2. for each $\epsilon>0$, there exists $\delta>0$ such that the $\left(x_{p}, x_{c}\right)$-component of any solution to the output feedback hybrid closed-loop system (13), (14) (resp. (8), (9)) starting from $\xi(0,0)=\left(\hat{x}_{p}(0,0), x_{c}(0,0), e(0,0), \tau(0,0)\right)=$ $\left(x_{p 0}, x_{c 0}, e_{0}, \tau_{0}\right)$ with $x_{p 0} \neq 0, \tau_{0} \geq \rho$ and $\left|e_{0}\right| \leq \delta\left|\left(x_{p 0}, x_{c 0}\right)\right|$ is $\epsilon\left|\left(x_{p 0}, x_{c 0}\right)\right|$-close to a solution of the state feedback hybrid closed loop starting from $\left(x_{p}(0,0), x_{c}(0,0)\right)=\left(x_{p 0}, x_{c 0}\right)$.

Paralleling the previous section, we focus now on the second state-feedback reset rule proposed in $[25$, Theorem 1] and we leave once again the dynamics of the controller (14) unchanged but we define different jump and flow sets, 
based on the pair of matrices $K_{p} \in \mathbb{R}^{p \times n_{p}}$ and $\bar{P}_{p} \in \mathbb{R}^{n_{p} \times n_{p}}, \bar{P}_{p}=\bar{P}_{p}^{T}>0$, satisfying Assumption 3. In particular, the jump and flow sets are defined as:

$$
\begin{aligned}
& \mathcal{F}_{y}=\left\{\zeta:\left[\begin{array}{c}
\hat{x}_{p} \\
x_{c}
\end{array}\right]^{T}\left(N_{p}+\left[\begin{array}{cc}
\tilde{\alpha} \bar{P}_{p} & 0 \\
0 & \epsilon I
\end{array}\right]\right)\left[\begin{array}{c}
\hat{x}_{p} \\
x_{c}
\end{array}\right] \leq\left[\begin{array}{c}
\hat{x}_{p} \\
x_{c} \\
\eta
\end{array}\right]^{T}\left[\begin{array}{c}
K_{x} \\
K_{c} \\
K_{\eta}
\end{array}\right] \eta\right\}, \\
& \mathcal{J}_{y}=\left\{\zeta:\left[\begin{array}{c}
\hat{x}_{p} \\
x_{c}
\end{array}\right]^{T}\left(N_{p}+\left[\begin{array}{cc}
\tilde{\alpha} \bar{P}_{p} & 0 \\
0 & \epsilon I
\end{array}\right]\right)\left[\begin{array}{c}
\hat{x}_{p} \\
x_{c}
\end{array}\right] \geq\left[\begin{array}{c}
\hat{x}_{p} \\
x_{c} \\
\eta
\end{array}\right]^{T}\left[\begin{array}{c}
K_{x} \\
K_{c} \\
K_{\eta}
\end{array}\right] \eta\right.
\end{aligned}
$$

where $\epsilon>0,0<\tilde{\alpha} \leq \alpha$ and $N_{p}$ and $\bar{P}_{p}$ are selected in the same way as in the previous section.

The closed-loop system (14), (15) generalizes to the output feedback case the state-feedback solution whose properties are established in [26, Theorem 1] (see also [25, Proposition 2]). The following theorem establishes the global exponential stability of the scheme.

Theorem 4 Consider a plant-controller pair (1), (12) satisfying Assumption 1, three parameters $\bar{P}_{p}, K_{p}$ and $\alpha$ satisfying Assumption 3 and five parameters $\epsilon>0, K_{y}, K_{x}, K_{c}, K_{\eta}$. Then there exists $\rho^{*}>0$ such that for all $\rho \in\left(0, \rho^{*}\right]$, the set $\mathcal{A}$ in (10) is globally exponentially stable for the closed-loop (14) with the sets $\mathcal{F}_{y}$ and $\mathcal{J}_{y}$ in $(15)$.

We cannot establish an equivalent statement to Proposition 1 with reference to the scheme (14), (15) and the linear state-feedback law of [25, Proposition 2] and [26]. Indeed, as emphasized at the end of Section 2.2, the jump and flow sets considered in [25-27] correspond to the ones in (15) with $e=0$ and $\epsilon=0$. Due to this fact, since we require $\epsilon>0$ here, we cannot say that the solutions to (14), (15) graphically converge to those of the corresponding state-feedback loops of [25-27]. Nevertheless, since the system with a small $\epsilon>0$ corresponds to a perturbation of the system with $\epsilon=0$, we can state by relying on the results of [17] that the arising trajectories can be made arbitrarily close to those of the state-feedback law of [25, Proposition 2] and [26] by choosing $\epsilon$ arbitrarily small. Note that the same consideration may be done on Theorem 2 and solutions of (9), (11). Nevertheless for both Theorems 3 and 4 we can state the following properties with respect to the results in [11] (by virtue of Remark 4 the same statement can be adapted to Theorems 1 and 2 by replacing (13), (14) and (14), (15) by (8), (9) and (9), (11), respectively). The proof of Proposition 2 is omitted because it is identical to the proof of Proposition 1.

Proposition 2 Consider the state feedback hybrid closed loops in [11, Theorem 1 and 2]. Then the following holds:

1. any solution of that state feedback hybrid closed loop in [11, Theorem 1] (resp. [11, Theorem 2]) starting from $\left(x_{p}(0,0), x_{c}(0,0), \tau(0,0)\right)=\left(x_{p 0}, x_{c 0}, \tau_{0}\right)$ is also a solution of the output feedback hybrid closed-loop system (13), (14) (resp. (14), (15)) starting from $\xi(0,0)=\left(\hat{x}_{p}(0,0), x_{c}(0,0), e(0,0), \tau(0,0)\right)=\left(x_{p 0}, x_{c 0}, 0, \tau_{0}\right)$;

2. for each $\epsilon>0$, there exists $\delta>0$ such that the $\left(x_{p}, x_{c}\right)$-component of any solution to the output feedback hybrid closed-loop system (13), (14) (resp. (14), (15)) starting from $\xi(0,0)=\left(\hat{x}_{p}(0,0), x_{c}(0,0), e(0,0), \tau(0,0)\right)=$ $\left(x_{p 0}, x_{c 0}, e_{0}, \tau_{0}\right)$ with $x_{p 0} \neq 0$ and $\left|e_{0}\right| \leq \delta\left|\left(x_{p 0}, x_{c 0}\right)\right|$ is $\epsilon\left|\left(x_{p 0}, x_{c 0}\right)\right|$-close to a solution of the state feedback hybrid closed loop starting from $\left(x_{p}(0,0), x_{c}(0,0), \tau(0,0)\right)=\left(x_{p 0}, x_{c 0}, \tau_{0}\right)$. 
Remark 3 In (14b) and in the sets (13) and (15), the matrices $K_{y}, K_{x}, K_{c}, K_{\eta}$ are completely free due to the cascaded structure of the scheme. In particular, since all these matrices are multiplied by $e$ or $\eta$ and $\operatorname{since} \lim _{t+j \rightarrow \infty} e(t, j)=0$ implies $\lim _{t+j \rightarrow \infty} \eta(t, j)=0$, these terms become ineffective once the error approaches to zero. Nevertheless, during the observer estimation transient, when $e \neq 0$ and possibly $\eta=\bar{C}_{p} e \neq 0$ as well, nonzero selections of these parameters can beneficially modify the transient response. For example, it may be useful to choose $K_{\eta}$ positive definite and possibly large as this will enlarge the flow set (see (13) and (15)) in the $\eta$ direction so that jumps will be inhibited when $\eta=\bar{C}_{p} e$ is large. This is reasonable because the jump map involves the estimated state and might (transiently) assign the controller state to an inaccurate value when the estimation error is large. On the other hand, the gain $K_{y}$ may affect in a beneficial way the jump map when the output error is nonzero and may be manually tuned by inspecting the closed-loop response when the error is large (it is recalled that these gains essentially have no effect after the estimation error becomes small). The potential behind these parameters $K_{\eta}, K_{y}$ is illustrated in the examples of Section 3, while the other parameters $K_{x}, K_{c}$ are selected to be zero since their impact during the observer transient remains unclear and no specific tuning rule is available.

Remark 4 System (9) with flow and jump sets (8) (resp. (11)) is equivalent to system (14) with flow and jump sets (13) (resp. (15)) with $K_{y}, K_{x}, K_{c}, K_{\eta}$ equal to zero. For this reason Theorems 1 and 2 are corollaries of Theorems 3 and 4. Therefore, we will only prove Theorems 3 and 4 in Section 4. Note also that, due to this fact, Propositions 1 and 2 also apply to the results of Theorems 1 and 2 . We also emphasize that the results of Section 2.2 , in addition to being more intuitive in the way they are presented, they also lead to a simpler implementation, indeed the flow and jump sets (8) and (11) are defined based on a smaller subset of parameters.

Remark 5 It is worth commenting on the difference between the dwell-time approach taken in this paper and the parallel results of [10] where no dwell time is used to address the extension of the results of [25-27] to the output feedback case. As already emphasized in the introduction, the main difficulty arising from this output feedback extension corresponds to the fact that the results in [25-27] exhibit Zeno solutions at the origin which, when extending the scheme to a feedback from the observed state may become non-converging Zeno solutions. Then the approach in [10] for suppressing the Zeno solutions was to remove a small ball around the origin (thereby resulting in global practical asymptotic stability). Here, instead, we use a dwell-time logic to remove the Zeno solutions and we are able to establish global exponential stability. Note that a similar distinction can be made between the work in [12] which establishes global practical asymptotic stability and its revised version in [13] where global exponential stability is obtained with a dwell-time logic.

\section{Simulations}

In this section, two simulation examples are presented to show the effectiveness of the proposed methods. For the techniques in Theorems 1 and 3, we use an example which appeared in several reset control papers. For the techniques in Theorems 2 and 4, we present an example inspired by a positioning system comprising an electrical DC motor. 


\subsection{Illustration of Theorems 1 and 3}

Consider the plant $P(s)=\frac{s+1}{s(s+0.2)}$ introduced in [3] and discussed in [10,22, 26]. According with (1), a possible realization is

$$
\left[\begin{array}{c|c}
\bar{A}_{p} & \bar{B}_{p} \\
\hline \bar{C}_{p} & \bar{D}_{p}
\end{array}\right]=\left[\begin{array}{cc|c}
-0.6 & 0.6 & -1 \\
-0.4 & 0.4 & 1 \\
\hline 0 & 1 & 0
\end{array}\right]
$$

Notice that the pair $\left(\bar{C}_{p}, \bar{A}_{p}\right)$ is observable. To design the continuous-time part (2a) of the controllers, we select the matrices $\bar{A}_{c}, \bar{B}_{c}, \bar{C}_{c}$ and $\bar{D}_{c}$ to define the same closed-loop used in [3,10,26], obtaining

$$
\left[\begin{array}{c|c}
\bar{A}_{c} & \bar{B}_{c} \\
\hline \bar{C}_{c} & \bar{D}_{c}
\end{array}\right]=\left[\begin{array}{c|c}
-1 & -1 \\
\hline 1 & 0
\end{array}\right],
$$

For the hybrid part of our controller, we exploit the optimal configuration presented for the static state feedback in [26] for the overshoot reduction, see Table 1. As the basic idea of this optimization is to approximate the Lyapunov-like function, used to define the flow and jump sets, to the norm of the plant output (i.e. $\left.V\left(x_{p}, x_{c}\right) \approx\left|y_{p}\right|^{2}\right)$, we select $P_{c}=10^{-10}$ (namely the smallest $P_{c}$ that satisfies all the conditions in Assumption 2) in such a way that the influence of the sub-state $x_{c}$ be reduced. Moreover we set $\tilde{\alpha}=10^{-8}$ to enlarge as much as possible the flow set and $\rho=2 \cdot 10^{-3}$. The observer gain in (2a) is chosen as $L=\left[\begin{array}{ll}0.26 & 1.37\end{array}\right]^{T}$ and, following the technique of Remark 3, the controller (14) is implemented with $K_{x}=0, K_{c}=0$, and $K_{\eta}=0$. As for $K_{y}$, the inspection of the transient response revealed that $K_{y}=-5$ leads to an improved transient, partially recovering the performance of the solid line obtained in [26] in

Table 1: Hybrid controller setting proposed

\begin{tabular}{|cc|c|c|}
\hline $\bar{P}_{p}$ & $K_{p}^{T}$ & $\alpha$ \\
\hline$\left[\begin{array}{cc}0.0097785 & -0.0096375 \\
-0.0096375 & 0.99990\end{array}\right]$ & {$\left[\begin{array}{c}0.0594992 \\
-4.83065\end{array}\right]$} & $7.784 \cdot 10^{-6}$ \\
\hline
\end{tabular}
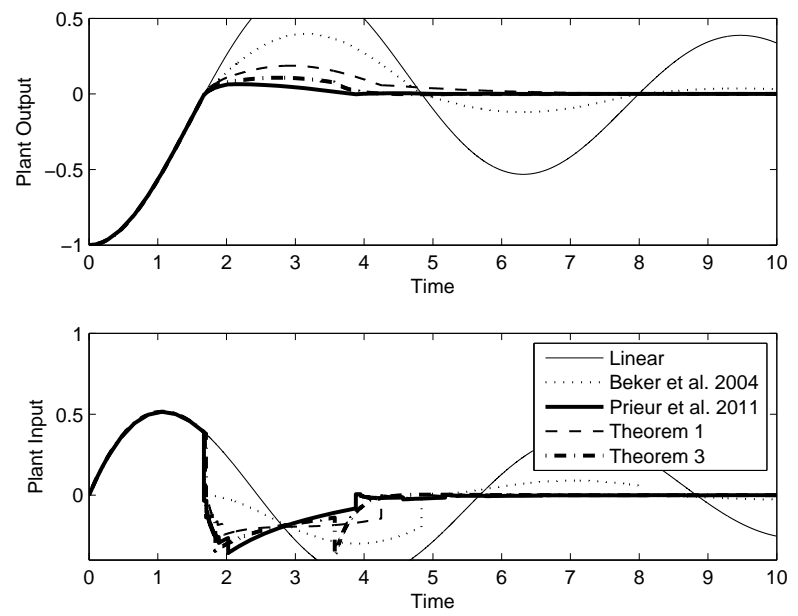

Figure 2: Hybrid controllers (7) and (12), compared to the linear case, to the FORE in [3] and to the hybrid controller with optimal static state feedback given in [26]. 
which the knowledge of the state was assumed.

Figure 2 depicts the input and output behavior of the hybrid systems (9) (dashed line) and (14) (dot-dashed line) compared to the linear case, to the technique in [3] and to the technique in [26]. All the controllers have zero initial conditions whereas the plant state starts from $x_{p}(0,0)=-\left[\begin{array}{ll}1 & 1\end{array}\right]^{T}$. As expected, the undesired effects of the dynamics of the observer affect the controller (7) exhibiting a larger overshoot than the static state feedback of [26]. This overshoot is caused by the observer transient. The second control technique is capable to partially compensate for this gap, recovering some performance for the output feedback case. We remark that, although the FORE in [3] does not introduce further dynamics for the hybrid closed-loop system, it exhibits a larger overshoot than (9) and (14).

\subsection{Illustration of Theorems 2 and 4}

Let us consider the use of a DC motor to place a load in a desired position (namely, the origin). Figure 3 represents the series of the DC motor and the load that we are considering, where $K_{e}=10$ and $\tau_{e}=0.05$ are the electrical gain and time constant of the motor, $F=1.2$ and $J=0.5$ are the friction and inertia of the load. Note that $J=0.5$ corresponds, for example, to the inertia of a cylindric load with radius $R=0.25 \mathrm{~m}$ and mass $m=16 \mathrm{Kg}$.

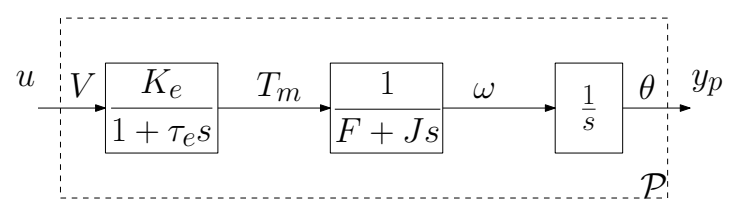

Figure 3: Plant block scheme.

With these parameters, the plant in observer canonical form is

$$
\left[\begin{array}{c|c}
\bar{A}_{p} & \bar{B}_{p} \\
\hline \bar{C}_{p} & \bar{D}_{p}
\end{array}\right]=\left[\begin{array}{ccc|c}
-22.4 & -6 & 0 & 50 \\
8 & 0 & 0 & 0 \\
0 & 1 & 0 & 0 \\
\hline 0 & 0 & 1 & 0
\end{array}\right],
$$

which can be commonly controlled by the following PI controller whose parameters have been tuned following a typical PI design procedure (see, e.g., [14, Chapter 4.3.4])

$$
\left[\begin{array}{c|c}
\bar{A}_{c} & \bar{B}_{c} \\
\hline \bar{C}_{c} & \bar{D}_{c}
\end{array}\right]=\left[\begin{array}{c|c}
0 & 0.1250 \\
\hline-0.08 & -0.05
\end{array}\right] .
$$

We want to augment this controller with a hybrid loop tuned such a way to reduce the overshoot induced by the integral action. Two different hybrid loops are presented: one with a full order observer (namely, an observer with the same order as the plant) and one with a second order observer (designed based on a second order approximate model of the plant). In both cases, the optimization technique in [26, Theorem 3] for the overshoot reduction is used. In all the plots, the linear PI controller will be compared to the results obtained with different settings of the same linear controller augmented by a hybrid loop.

Another aspect that will be taken into account is the robustness to parameters uncertainty. To this aim, we will consider the case in which the load has a mass $100 \%$ higher than expected, therefore the perturbed inertia value is 
$J=1$ (see Figure 3) and the perturbed plant matrices become

$$
\left[\begin{array}{c|c}
\bar{A}_{p} & \bar{B}_{p} \\
\hline \bar{C}_{p} & \bar{D}_{p}
\end{array}\right]=\left[\begin{array}{ccc|c}
-21.2 & -6 & 0 & 50 \\
4 & 0 & 0 & 0 \\
0 & 1 & 0 & 0 \\
\hline 0 & 0 & 1 & 0
\end{array}\right] .
$$

\subsubsection{Hybrid loop based on a full order observer}

Let us consider the technique in [26, Theorem 3], for $\kappa_{M}=0.1$ (with the notation of [26]) and $L=\left[\begin{array}{lll}-0.0241 & 0.0841 & 1.0808\end{array}\right]^{T}$, we get (according to (5) and (6)):

$$
\begin{aligned}
K_{p}= & {\left[\begin{array}{lll}
0.00803345 & 0.02347159 & 0.09657843
\end{array}\right], } \\
\rho_{y}= & 0.2809, \\
\bar{P}_{p}= & {\left[\begin{array}{lll}
0.02290376 & 0.06418020 & 0.06227308 \\
0.06418020 & 0.19173178 & 0.18092975 \\
0.06227308 & 0.18092975 & 0.95345625
\end{array}\right], }
\end{aligned}
$$

where $\rho_{y}$ is the quantity to be minimized (according to [26]). Then, by selecting $\tilde{\alpha}=10^{-12}, \rho=0.004, \epsilon=0.01$ and $N_{p}$ as in (11c), it is possible to define system (9) with the flow and jump sets in (11).

In all of the next simulations, the plant and controller initial conditions will be $x_{p}(0,0)=[0.70-4]^{T}, x_{c}(0,0)=0$ and $\tau(0,0)=\rho$, whereas the observer initial condition is modified to explore the behavior of the hybrid controller. Choosing $\hat{x}_{p}(0,0)=x_{p}(0,0)$ (namely, the estimate error is zero), the output of the hybrid closed-loop system and the output of the linear closed loop with the simple PI are plotted in Figure 4(a) for the nominal case. Note that with a full order observer if $e(T)=0$ then $e(t)=0$ for all $t \geq T$ (in the absence of disturbances).

Figure 4(b) compares the behavior of the PI and the hybrid controllers with the plant subject to uncertainties of the parameters. The hybrid controller behavior is illustrated in several scenarios to better show how it is possible
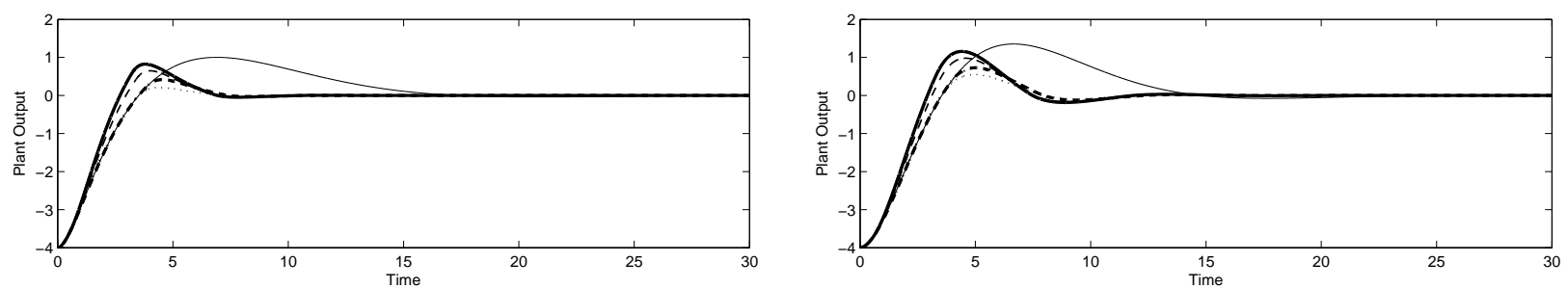

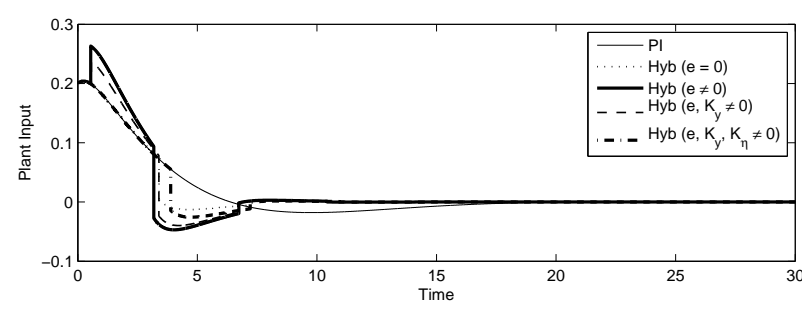

(a) Nominal case.

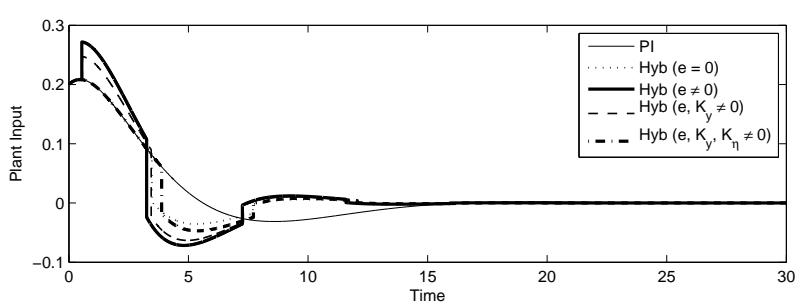

(b) Perturbed case.

Figure 4: Full order observer case (Example of Section 3.2.1). 

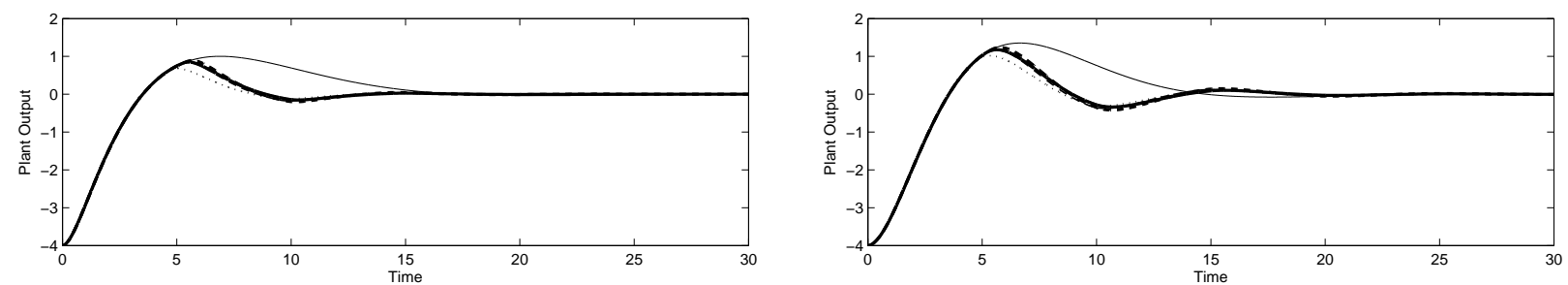

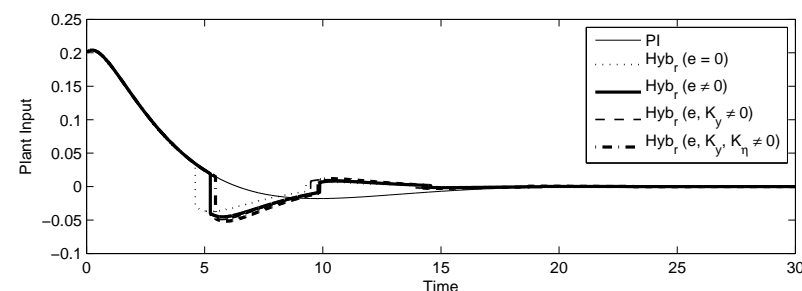

(a) Nominal case.

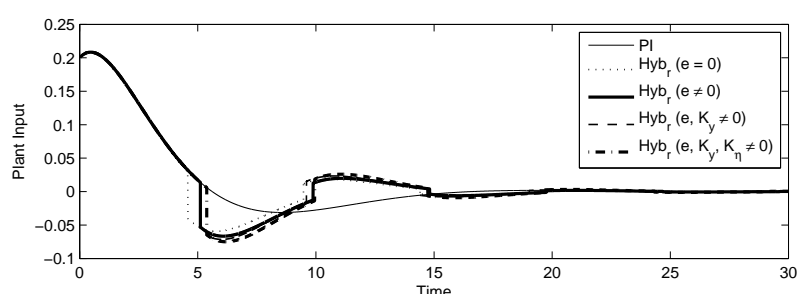

(b) Perturbed case.

Figure 5: Approximated reduced order observer case (Example of Section 3.2.2).

to compensate for the undesired effects caused by a non zero observation error. The overshoot reduction for the hybrid system is maintained in the case with parameters uncertainties when the estimate error of the observer is zero (dash-dotted line), but it may be lost if $e$ is large (dashed line). We remark that the faster rise time in the dashed line is not necessarily more desirable since it requires a larger control input (see lower plot). To compensate for this overshoot, we resort to the hybrid controller in (14) (same settings as for (9), plus a further term $K_{y}=0.06$ in the reset law) maintaining the flow and jump sets unchanged (namely, we select the sets in $(15)$ with $K_{x}, K_{c}, K_{\eta}$ all zero). In this way we reduce the overshoot but the system still shows a faster rise time (dotted line). Finally, we introduce the gain $K_{\eta}=0.2$, which inhibits jumps when the output estimate error is large as discussed in Remark 3 . The resulting response, corresponding to the thin solid line, appears to be closer to the state-feedback response.

\subsubsection{Hybrid loop based on an approximate reduced order observer}

Let us consider the plant of Figure 3 neglecting the first block, related to the electrical dynamics of the DC motor. Then the remaining part is

$$
\left[\begin{array}{c|c}
\bar{A}_{p r} & \bar{B}_{p r} \\
\hline \bar{C}_{p r} & \bar{D}_{p r}
\end{array}\right]=\left[\begin{array}{rr|r}
-2.4 & 0 & 2 \\
1 & 0 & 0 \\
\hline 0 & 1 & 0
\end{array}\right],
$$

and it can be exploited to define a reduced order observer (the subscript "r" stands for "reduced"). Since the goal is the overshoot reduction, we use the technique in [26, Theorem 3], with $\kappa_{M}=0.1$ (see [26]) and $L=[0.00630 .9015]^{T}$, to obtain (according to $(5)$ and $(6)$ )

$$
\begin{aligned}
K_{p} & =\left[\begin{array}{ll}
0.03680221 & 0.09245721
\end{array}\right], \\
\rho_{y} & =0.3435, \\
\bar{P}_{p} & =\left[\begin{array}{ll}
0.41279516 & 0.24891952 \\
0.24891952 & 0.89441164
\end{array}\right],
\end{aligned}
$$


where $\rho_{y}$ is the quantity to be minimized (according to [26]). Then, by selecting $\tilde{\alpha}=10^{-12}, \rho=0.004, \epsilon=0.01$, $K_{y}=0.06, K_{x}=0, K_{c}=0, K_{\eta}=0.2$ and $N_{p}$ as in (11c), it is possible to define system (9) with the flow and jump sets in (11).

Figure 5 shows that the behavior of the hybrid closed-loop system does not change much compared to the full order case. The overshoot reduction is achieved and no faster rise time or other considerable effects coming from the observer can be observed, even for very large initial estimate errors.

\section{Proofs of the main results}

As pointed out in Remark 4, Theorem 1 is a corollary of Theorem 3 and Theorem 2 is a corollary of Theorem 4, all of them corresponding to the special case of selecting zero matrices for $K_{y}, K_{x}, K_{c}$ and $K_{\eta}$. As a consequence, we only prove next Theorems 3 and 4 and Proposition 1.

Proof of Theorem 3. As a first step, consider the following change of coordinates: $x=\left[\hat{x}_{p}^{T} x_{c}^{T} e^{T}\right]^{T} \mapsto \bar{x}:=$ $\left[\hat{x}_{p}^{T} \sigma^{T} e^{T}\right]^{T}:=\left[\hat{x}_{p}^{T}\left(x_{c}-K_{p} \hat{x}_{p}-K_{y} \bar{C}_{p} e\right)^{T} e^{T}\right]^{T}$. In this transformed set of coordinates, it can be verified that the flow dynamics in (14) corresponds to (see also (3)):

$$
\left[\begin{array}{c}
\dot{x}_{p} \\
\dot{\sigma} \\
\dot{e}
\end{array}\right]=\left[\begin{array}{ccc}
A_{p}+B_{p} K_{p} & A_{12} & A_{13} \\
A_{21} & A_{22} & A_{23} \\
0 & 0 & A_{e}
\end{array}\right]\left[\begin{array}{c}
\hat{x}_{p} \\
\sigma \\
e
\end{array}\right],
$$

where $A_{12}:=B_{p}, A_{13}:=B_{o}+B_{p} K_{y} \bar{C}_{p}, A_{21}:=B_{c}-K_{p}\left(A_{p}+B_{p} K_{p}\right)+A_{c} K_{p}, A_{22}:=A_{c}-K_{p} B_{p}$ and $A_{23}:=$ $A_{c} K_{y} \bar{C}_{p}-K_{y} \bar{C}_{p} A_{e}+B_{c}-K_{p}\left(B_{o}+B_{p} K_{y} \bar{C}_{p}\right)$ are constant matrices.

Then, using the identities in (6), it can be verified after some calculations that

$$
\begin{aligned}
\hat{x}_{p}^{T} \bar{P}_{p} \hat{x}_{p}+\sigma^{T} P_{c} \sigma & =\left[\begin{array}{c}
\hat{x}_{p} \\
x_{c}
\end{array}\right]^{T} P\left[\begin{array}{c}
\hat{x}_{p} \\
x_{c}
\end{array}\right]+2\left[\begin{array}{c}
\hat{x}_{p} \\
x_{c} \\
e
\end{array}\right]^{T}\left[\begin{array}{c}
\Sigma_{13} \\
\Sigma_{23} \\
\Sigma_{33}
\end{array}\right] e \\
& =V\left(\hat{x}_{p}, x_{c}\right)+2\left[\begin{array}{c}
\hat{x}_{p} \\
x_{c} \\
e
\end{array}\right]^{T} \Sigma e,
\end{aligned}
$$

where $\Sigma_{13}:=K_{p}^{T} P_{c} K_{y} \bar{C}_{p}, \Sigma_{23}:=-P_{c} K_{y} \bar{C}_{p}$ and $\Sigma_{33}:=\frac{1}{2} \bar{C}_{p}^{T} K_{y}^{T} P_{c} K_{y} \bar{C}_{p}$ are constant matrices.

Let us now introduce the function $\varphi(\tau):=\exp ((2 \rho-\tau) \lambda)$, where $\lambda>0$ is a scalar to be selected later and note that, for all $\tau \in[0,2 \rho]$, this function satisfies:

$$
\begin{aligned}
& 1 \leq \varphi(\tau) \leq \exp (2 \lambda \rho) \\
& \dot{\varphi}(\tau)=-\lambda \dot{\tau} \varphi(\tau) \leq 0 .
\end{aligned}
$$

Based on this equation, we consider the following positive definite and radially unbounded candidate Lyapunov function:

$$
W_{\xi}(\bar{x}, \tau):=\hat{x}_{p}^{T} \bar{P}_{p} \hat{x}_{p}+\varphi(\tau) \sigma^{T} P_{c} \sigma+\xi e^{T} P_{e} e
$$


where $\bar{P}_{p}$ and $P_{c}$ are defined in Assumption 2 (see also (6)), $\xi>0$ is a scalar (to be selected later) and $P_{e}=P_{e}^{T}>0$ is the solution of the Lyapunov equation $A_{e}^{T} P_{e}+P_{e} A_{e}=-I$. Note that $P_{e}$ always exists because $A_{e}$ is Hurwitz by Assumption 1. From (18) it follows that for all $\bar{x} \in \mathbb{R}^{2 n_{p}+n_{c}}$ and for all $\tau \in[0,2 \rho]$,

$$
c_{1}|\bar{x}|^{2} \leq W_{\xi}(\bar{x}, \tau) \leq c_{2}|\bar{x}|^{2}
$$

where $c_{1}=\min \left\{\lambda_{\min }\left(\bar{P}_{p}\right), \lambda_{\min }\left(P_{c}\right), \xi \lambda_{\min }\left(P_{e}\right)\right\}$ and $c_{2}=\max \left\{\lambda_{\max }\left(\bar{P}_{p}\right), \exp (2 \lambda \rho) \lambda_{\max }\left(P_{c}\right), \xi \lambda_{\max }\left(P_{e}\right)\right\}$.

To complete the proof of the theorem, we will make use of the following claim, whose proof is reported next.

Claim 1 There exists a small enough $\rho^{*}$ and a large enough $\xi^{*}$ such that for all $\rho \leq \rho^{*}$ and all $\xi \geq \xi^{*}$ the function $W_{\xi}$ in (19) satisfies, for some $\gamma>0$,

$$
\begin{array}{ll}
\dot{W}_{\xi}(\bar{x}, \tau)<-\gamma|\bar{x}|^{2} & \text { if } T_{\zeta} \bar{x} \in \mathcal{F}_{y} \backslash\{0\} \text { or } \tau \in[0, \rho] \\
\Delta W_{\xi}(\bar{x}, \tau) \leq 0 & \text { if } T_{\zeta} \bar{x} \in \mathcal{J}_{y} \backslash\{0\} \text { and } \tau \in[\rho, 2 \rho],
\end{array}
$$

where $\Delta W_{\xi}(\bar{x}, \tau):=W_{\xi}\left(\bar{x}^{+}, \tau^{+}\right)-W_{\xi}(\bar{x}, \tau)$ and $T_{\zeta}:=\left[\begin{array}{ccc}I & 0 & 0 \\ 0 & I & 0 \\ 0 & 0 & C_{p}\end{array}\right] \bar{T}:=\left[\begin{array}{ccc}I & 0 & 0 \\ 0 & I & 0 \\ 0 & 0 & C_{p}\end{array}\right]\left[\begin{array}{ccc}I & 0 & 0 \\ K_{p} & I & K_{y} \\ 0 & 0 & I\end{array}\right]$ satisfies $\zeta=T_{\zeta} \bar{x}$.

From the properties (20) and (21), we can apply [28, Theorem 7.6] to establish that the set $\mathcal{A}$ in (10) is stable. Moreover, exponential stability of the set $\mathcal{A}$ comes from [32, Theorem 2] where the nonstrict inequality in (21b) is dealt with by exploiting the dwell-time property of temporally regularized solutions. In particular, by decomposing the closed-loop state in its components $\bar{x}$ and $\tau$, conditions 1)-4) of [32, Assumption 1] are satisfied due to (20) and (21) and because the pair $\left(\epsilon I_{n}, A\right)$ (where $A$ is defined in (3a)) is trivially observable. Finally, condition 5 ) of [32, Assumption 1] is satisfied because the hybrid solutions obey the dwell-time constraint. Thus, $\mathcal{A}$ is globally exponentially stable.

Proof of Claim 1 Equation (21b) follows trivially from noticing that the jump rule in (14) can be written as $\bar{x}^{+}=\left[\hat{x}_{p}^{+T} \sigma^{+T} e^{+T}\right]^{T}=\left[\hat{x}_{p}^{T} 0^{T} e^{T}\right]^{T}$ and due to the block diagonal structure of $W_{\xi}$ in (19) we have $W_{\xi}^{+} \leq W_{\xi}$ everywhere, because the second term becomes zero after the jump and the other terms remain unchanged.

To prove equation (21a) we separate the analysis in two cases, corresponding to the two flow conditions appearing in $(21 a)$.

Case 1: $\tau \in[0, \rho]$.

From the special structure of the dynamics in (16) and since equation (5) holds from Assumption 2, then, also using the inequalities in (18) and the fact that $\dot{\tau}=1$ whenever $\tau \in[0, \rho]$, the time derivative of $W_{\xi}$ in (19) satisfies

$$
\begin{aligned}
& \dot{W}_{\xi}(\bar{x}, \tau)=2 \hat{x}_{p}^{T} \bar{P}_{p}\left(A_{p}+B_{p} K_{p}\right) \hat{x}_{p}+2 \hat{x}_{p}^{T} \bar{P}_{p}\left(A_{12} \sigma+A_{13} e\right)-\lambda \varphi(\tau) \sigma^{T} P_{c} \sigma+2 \varphi(\tau) \sigma^{T} P_{c} \dot{\sigma}+2 \xi e^{T} P_{e} A_{e} e \\
& \leq-\tilde{\alpha} \hat{x}_{p}^{T} \bar{P}_{p} \hat{x}_{p}-\lambda \sigma^{T} P_{c} \sigma-\xi e^{T} e+2\left[\begin{array}{c}
\hat{x}_{p} \\
\sigma \\
e
\end{array}\right]^{T}\left[\begin{array}{ccc}
0 & \bar{P}_{p} A_{12} & \bar{P}_{p} A_{13} \\
\varphi(\tau) P_{c} A_{21} & \varphi(\tau) P_{c} A_{22} & \varphi(\tau) P_{c} A_{23} \\
0 & 0 & 0
\end{array}\right]\left[\begin{array}{c}
\hat{x}_{p} \\
\sigma \\
e
\end{array}\right] \text {, }
\end{aligned}
$$

where $A_{i j}, i=1, \ldots, 2, j=1, \ldots, 3$ are taken from (16). Note that the first three terms of the last equation are negative definite quadratic terms and both $\xi$ and $\lambda$ can be selected arbitrarily large to complete squares with the mixed terms arising from the fourth term. Moreover, from the first equation in $(18), \varphi(\tau)$ is bounded from above and 
from below and, once $\lambda$ has been fixed large enough, the upper bound on $\varphi(\tau)$ can be made arbitrarily close to 1 for all $\rho \leq \rho_{1}^{*}$, as long as $\rho_{1}^{*}>0$ is selected small enough. In this way, the terms in which $\varphi(\tau)$ figures can be maintained small. Then, there exist large enough selections of $\lambda$ and $\xi_{1}$ and a small enough $\rho_{1}^{*}$ such that for all $\rho \in\left[0, \rho_{1}^{*}\right]$ and all $\xi \geq \xi_{1}$, equation (22) implies

$$
\dot{W}_{\xi}(\bar{x}, \tau) \leq-\frac{\tilde{\alpha}}{2}|\bar{x}|^{2}, \quad \forall \bar{x} \in \mathbb{R}^{2 n_{p}+n_{c}}, \forall \tau \in[0, \rho] .
$$

Case 2: $T_{\zeta} \bar{x} \in \mathcal{F}_{y}$.

First note that from the definition of $V$, from (3a) and the definition of $N$ in (8c), we get

$$
\dot{V}\left(\hat{x}_{p}, x_{c}\right)=\left[\begin{array}{c}
\hat{x}_{p} \\
x_{c}
\end{array}\right]^{T} N\left[\begin{array}{l}
\hat{x}_{p} \\
x_{c}
\end{array}\right]+2\left[\begin{array}{c}
\hat{x}_{p} \\
x_{c}
\end{array}\right]^{T} P\left[\begin{array}{c}
B_{o} \\
B_{c}
\end{array}\right] e,
$$

Using (17), we can rewrite the function $W_{\xi}$ in (19) as follows

$$
\begin{aligned}
W_{\xi}(\bar{x}, \tau) & =\hat{x}_{p}^{T} \bar{P}_{p} \hat{x}_{p}+\sigma^{T} P_{c} \sigma+(\varphi(\tau)-1) \sigma^{T} P_{c} \sigma+\xi e^{T} P_{e} e \\
& =V\left(\hat{x}_{p}, x_{c}\right)+2\left[\begin{array}{c}
\hat{x}_{p} \\
x_{c} \\
e
\end{array}\right]^{T} \Sigma e+(\varphi(\tau)-1) \sigma^{T} P_{c} \sigma+\xi e^{T} P_{e} e
\end{aligned}
$$

Therefore, using (23), the flow set definition in (13a), the first equation in (18) and the second equation in (18), we get

$$
\begin{aligned}
\dot{W}_{\xi}(\bar{x}, \tau)= & {\left[\begin{array}{c}
\hat{x}_{p} \\
x_{c}
\end{array}\right]^{T} N\left[\begin{array}{l}
\hat{x}_{p} \\
x_{c}
\end{array}\right]+2\left[\begin{array}{c}
\hat{x}_{p} \\
x_{c} \\
e
\end{array}\right]^{T}\left(\Sigma A_{e}+\left[\begin{array}{c}
P\left[\begin{array}{c}
B_{o} \\
B_{c}
\end{array}\right] \\
0
\end{array}\right]+A^{T} \Sigma\right) e } \\
& -\lambda \dot{\tau} \varphi(\tau) \sigma^{T} P_{c} \sigma+2(\varphi(\tau)-1) \sigma^{T} P_{c} \dot{\sigma}+2 \xi e^{T} P_{e} \dot{e} \\
\leq & -\tilde{\alpha}\left[\begin{array}{c}
\hat{x}_{p} \\
x_{c}
\end{array}\right]^{T} P\left[\begin{array}{c}
\hat{x}_{p} \\
x_{c}
\end{array}\right]+2\left[\begin{array}{c}
\hat{x}_{p} \\
x_{c} \\
e
\end{array}\right]^{T}\left(\Sigma A_{e}+\left[\begin{array}{c}
P\left[\begin{array}{c}
B_{o} \\
B_{c}
\end{array}\right] \\
0
\end{array}\right]+A^{T} \Sigma+\frac{1}{2}\left[\begin{array}{c}
K_{x} \bar{C}_{p} \\
K_{c} \bar{C}_{p} \\
\bar{C}_{p}^{T} K_{\eta} \bar{C}_{p}
\end{array}\right]\right) \\
& +2(\varphi(\tau)-1) \sigma^{T} P_{c} \dot{\sigma}-\xi|e|^{2} \\
= & -\tilde{\alpha}\left[\begin{array}{c}
\hat{x}_{p} \\
x_{c}
\end{array}\right]^{T} P\left[\begin{array}{c}
\hat{x}_{p} \\
x_{c}
\end{array}\right]+2\left[\begin{array}{c}
\hat{x}_{p} \\
x_{c} \\
e
\end{array}\right]^{T}\left[\begin{array}{c}
Z_{13} \\
Z_{23} \\
Z_{33}
\end{array}\right] e+\kappa(\tau, \rho) x^{T} \Xi x-\xi|e|^{2},
\end{aligned}
$$

where $Z_{i 3} i=1, \ldots, 3$ are suitable matrices, $\Xi:=\bar{T}^{-T}\left[\begin{array}{ccc}0 & 0 \\ P_{c} A_{21} & P_{c} A_{22} & P_{c} A_{23} \\ 0 & 0 & 0\end{array}\right] \bar{T}^{-1}$ is defined in such a way that $x^{T} \Xi x=$ $\sigma^{T} P_{c} \dot{\sigma}$ and, from (18), $\kappa(\tau, \rho)=2(\varphi(\tau)-1)$ satisfies $0 \leq \kappa(\tau, \rho) \leq 2(\exp (2 \rho \lambda)-1)$, namely for any fixed value of $\lambda$, $\kappa(\tau, \rho)$ can be made arbitrarily small by selecting $\rho$ sufficiently small. Due to this fact, it follows that, once $\lambda$ has been fixed according to Case 1 above, it is possible to select $\rho_{2}^{*}$ sufficiently small and $\xi_{2}$ sufficiently large such that for all $\rho \in\left[0, \rho_{2}^{*}\right]$, and $\xi \geq \xi_{2}$, inequality (24) implies

$$
\dot{W}_{\xi}(\bar{x}, \tau) \leq-\frac{\tilde{\alpha}}{2} \lambda_{\min }(P)|x|^{2}, \quad \forall x \text { s.t. }\left[\begin{array}{ccc}
I & 0 & 0 \\
0 & I & 0 \\
0 & 0 & C_{p}
\end{array}\right] x \in \mathcal{F}_{y}, \forall \tau \in[0,2 \rho] .
$$


Finally, combining the studies in Cases 1 and 2 above, the proof of $(21 \mathrm{a})$ follows from picking $\rho^{*}=\min \left\{\rho_{1}^{*}, \rho_{2}^{*}\right\}$, $\xi=\max \left\{\xi_{1}, \xi_{2}\right\}$ and $\gamma=\frac{\tilde{\alpha}}{2} \min \left\{1, \lambda_{\min }(P) \lambda_{\min }\left(T_{\zeta}^{T} T_{\zeta}\right)\right\}$.

Proof of Theorem 4. Consider (16) and (18) and the following positive definite and radially unbounded Lyapunov function candidate

$$
\bar{W}_{\xi_{\sigma}, \xi_{e}}(\bar{x}, \tau):=\hat{x}_{p}^{T} \bar{P}_{p} \hat{x}_{p}+\xi_{\sigma} \varphi(\tau) \sigma^{T} \sigma+\xi_{e} e^{T} P_{e} e
$$

where $\xi_{\sigma}>0$ and $\xi_{e}>0$ are scalars (to be selected later) and $P_{e}=P_{e}^{T}>0$ is the solution of the Lyapunov equation $A_{e}^{T} P_{e}+P_{e} A_{e}=-I . P_{e}$ always exists by Assumption 1 stating that $A_{e}$ is Hurwitz. Note that from (18), it follows that for all $\bar{x} \in \mathbb{R}^{2 n_{p}+n_{c}}$ and for all $\tau \in[0,2 \rho]$,

$$
c_{1}|\bar{x}|^{2} \leq \bar{W}_{\xi_{\sigma}, \xi_{e}}(\bar{x}, \tau) \leq c_{2}|\bar{x}|^{2}
$$

where $c_{1}=\min \left\{\lambda_{\min }\left(\bar{P}_{p}\right), \xi_{\sigma}, \xi_{e} \lambda_{\min }\left(P_{e}\right)\right\}$ and $c_{2}=\max \left\{\lambda_{\max }\left(\bar{P}_{p}\right), \xi_{\sigma} \exp (2 \lambda \rho), \xi_{e} \lambda_{\max }\left(P_{e}\right)\right\}$.

The proof of the theorem is completed by following the same steps of the end of the proof of Theorem 3 , using the following claim, which corresponds to Claim 1 rewritten for the case addressed here.

Claim 2 There exist positive numbers $\lambda, \xi_{\sigma}$ and $\xi_{e}$, and a small enough $\rho^{*}$ such that for all $\rho \leq \rho^{*}$ the function $\bar{W}_{\xi_{\sigma}, \xi_{e}}$ in (25) satisfies, for some $\gamma>0$,

$$
\begin{array}{ll}
\dot{\bar{W}}_{\xi_{\sigma}, \xi_{e}}(\bar{x}, \tau)<-\gamma|\bar{x}|^{2} & \text { if } T_{\zeta} \bar{x} \in \mathcal{F}_{y} \backslash\{0\} \text { or } \tau \in[0, \rho] \\
\Delta \bar{W}_{\xi_{\sigma}, \xi_{e}}(\bar{x}, \tau) \leq 0 & \text { if } T_{\zeta} \bar{x} \in \mathcal{J}_{y} \backslash\{0\} \text { and } \tau \in[\rho, 2 \rho],
\end{array}
$$

where $\Delta \bar{W}_{\xi_{\sigma}, \xi_{e}}(\bar{x}, \tau):=\bar{W}_{\xi_{\sigma}, \xi_{e}}\left(\bar{x}^{+}, \tau^{+}\right)-\bar{W}_{\xi_{\sigma}, \xi_{e}}(\bar{x}, \tau)$ and $T_{\zeta}:=\left[\begin{array}{ccc}I & 0 & 0 \\ 0 & I & 0 \\ 0 & 0 & \bar{C} \\ 0\end{array}\right] \bar{T}:=\left[\begin{array}{ccc}I & 0 & 0 \\ 0 & I & 0 \\ 0 & 0 & \bar{C}_{p}\end{array}\right]\left[\begin{array}{ccc}I & 0 \\ K_{p} & I & 0 \\ 0 & K_{y} \bar{C}_{p} \\ I\end{array}\right]$ satisfies $\zeta=T_{\zeta} \bar{x}$.

Proof of Claim 2 Equation (27b) follows trivially from noticing that the jump rule in (14) can be written as $\bar{x}^{+}=\left[\hat{x}_{p}^{+T} \sigma^{+T} e^{+T}\right]^{T}=\left[\hat{x}_{p}^{T} 0^{T} e^{T}\right]^{T}$ and due to the block diagonal structure of $\bar{W}_{\xi_{\sigma}, \xi_{e}}$ in $(25)$ we have $\bar{W}_{\xi_{\sigma}, \xi_{e}}^{+} \leq \bar{W}_{\xi_{\sigma}, \xi_{e}}$ everywhere, because the second term becomes zero after the jump and the other terms remain unchanged.

To prove equation (27a), we compute the derivative of $\bar{W}_{\xi_{\sigma}, \xi_{e}}$ in two cases, corresponding to the two flow conditions appearing in (27a). Then, we will combine the analysis of such cases to guarantee (27a).

Case 1: $\tau \in[0, \rho]$.

From the special structure of the dynamics in (16) and since equation (5) holds from Assumption 2, then, also using the inequalities in (18) and the fact that $\dot{\tau}=1$ whenever $\tau \in[0, \rho]$, the time derivative of $\bar{W}_{\xi_{\sigma}, \xi_{e}}$ in $(25)$ satisfies

$$
\begin{aligned}
\dot{\bar{W}}_{\xi_{\sigma}, \xi_{e}}(\bar{x}, \tau) & =2 \hat{x}_{p}^{T} \bar{P}_{p}\left(A_{p}+B_{p} K_{p}\right) \hat{x}_{p}+2 \hat{x}_{p}^{T} \bar{P}_{p}\left(A_{12} \sigma+A_{13} e\right)-\lambda \xi_{\sigma} \varphi(\tau) \sigma^{T} \sigma+2 \xi_{\sigma} \varphi(\tau) \sigma^{T} \dot{\sigma}+2 \xi_{e} e^{T} P_{e} A_{e} e \\
& \leq-\tilde{\alpha} \hat{x}_{p}^{T} \bar{P}_{p} \hat{x}_{p}-\lambda \xi_{\sigma} \sigma^{T} \sigma-\xi_{e} e^{T} e+2\left[\begin{array}{c}
\hat{x}_{p} \\
\sigma \\
e
\end{array}\right]^{T}\left[\begin{array}{ccc}
0 & \bar{P}_{p} A_{12} & \bar{P}_{p} A_{13} \\
\varphi(\tau) \xi_{\sigma} A_{21} & \varphi(\tau) \xi_{\sigma} A_{22} & \varphi(\tau) \xi_{\sigma} A_{23} \\
0 & 0 & 0
\end{array}\right]\left[\begin{array}{c}
\hat{x}_{p} \\
\sigma \\
e
\end{array}\right](28)
\end{aligned}
$$

where $A_{i j}, i=1, \ldots, 2, j=1, \ldots, 3$ are taken from (16). Note that the first three terms of the last equation are negative and that $\xi_{e}, \lambda \xi_{\sigma}$ can be adjusted by choosing $\xi_{\sigma}, \xi_{e}$ and $\lambda$ in $(25)$. In particular, since from $(18) 1 \leq \varphi(\tau) \leq \exp (2 \lambda \rho)$, 
then there exist positive numbers $c_{\sigma 1}, c_{\rho 1}$ and $\xi_{e 1}$ such that if

$$
\lambda \xi_{\sigma} \geq c_{\sigma 1}, \quad \lambda \rho \leq c_{\rho 1}, \quad \xi_{e} \geq \xi_{e 1}
$$

then (28) implies:

$$
\dot{\bar{W}}_{\xi_{\sigma}, \xi_{e}}(\bar{x}, \tau) \leq-\frac{\tilde{\alpha}}{2}|\bar{x}|^{2}, \quad \forall \bar{x} \in \mathbb{R}^{2 n_{p}+n_{c}}, \forall \tau \in[0, \rho]
$$

Case 2: $T_{\zeta} \bar{x} \in \mathcal{F}_{y}$.

Using the flow set definition in (15a) and the equations in (18), we get for all $T_{\zeta} \bar{x}=x \in \mathcal{F}_{y}$,

$$
\begin{aligned}
\dot{\bar{W}}_{\xi_{\sigma}, \xi_{e}}(\bar{x}, \tau)= & 2 \hat{x}_{p}^{T} \bar{P}_{p}\left(A_{p} \hat{x}_{p}+B_{p} x_{c}\right)+2 \hat{x}_{p}^{T} \bar{P}_{p} B_{o} e-\dot{\tau} \lambda \xi_{\sigma} \varphi(\tau) \sigma^{T} \sigma+2 \xi_{\sigma} \varphi(\tau) \sigma^{T} \dot{\sigma}+2 \xi_{e} e^{T} P_{e} A_{e} e \\
\leq & {\left[\begin{array}{c}
\hat{x}_{p} \\
x_{c}
\end{array}\right]^{T}\left[\begin{array}{cc}
-\tilde{\alpha} \bar{P}_{p} & 0 \\
0 & -\epsilon I
\end{array}\right]\left[\begin{array}{c}
\hat{x}_{p} \\
x_{c}
\end{array}\right]+\left[\begin{array}{c}
\hat{x}_{p} \\
x_{c} \\
e
\end{array}\right]^{T}\left[\begin{array}{c}
K_{x} \bar{C}_{p} \\
K_{c} \bar{C}_{p} \\
\bar{C}_{p}^{T} K_{\eta} \bar{C}_{p}
\end{array}\right] e+2 \hat{x}_{p}^{T} \bar{P}_{p} B_{o} e } \\
& +2 \xi_{\sigma} \varphi(\tau) \sigma^{T} \dot{\sigma}-\xi_{e}|e|^{2} \\
= & {\left.\left[\begin{array}{c}
\hat{x}_{p} \\
x_{c}
\end{array}\right]^{T}\left[\begin{array}{cc}
-\tilde{\alpha} \bar{P}_{p} & 0 \\
0 & -\epsilon I
\end{array}\right]\left[\begin{array}{c}
\hat{x}_{p} \\
x_{c}
\end{array}\right]+2 \xi_{\sigma} \varphi(\tau) x^{T} \Xi x+\left[\begin{array}{c}
\hat{x}_{p} \\
x_{c} \\
e
\end{array}\right] \begin{array}{c}
Z_{13} \\
Z_{23} \\
Z_{33}
\end{array}\right] e-\xi_{e}|e|^{2}, }
\end{aligned}
$$

where $Z_{i 3} i=1, \ldots, 3$ are suitable matrices, $\Xi:=\bar{T}^{-T}\left[\begin{array}{ccc}0 & 0 & 0 \\ A_{21} & A_{22} & A_{23} \\ 0 & 0 & 0\end{array}\right] \bar{T}^{-1}$ is defined in such a way that $x^{T} \Xi x=\sigma^{T} \dot{\sigma}$. At the right hand side of (30) we find a first term providing good quadratic decrease in $\hat{x}_{p}$ and $x_{c}$, followed by a second bad term which can be made arbitrarily small by choosing $\xi_{\sigma}$ (and $\lambda \rho$ too, due to the term $\left.\varphi(\tau)-\operatorname{see}(18)\right)$ small enough, followed by two terms providing mixed and quadratic terms in $e$ which can be dominated by selecting $\xi_{e}$ large enough. In particular, since $\varphi(\cdot)$ is bounded (see (18)), there exist positive numbers $c_{\sigma 2}, c_{\rho 2}$ and $\xi_{e 2}$ such that if

$$
\xi_{\sigma} \leq c_{\sigma 2}, \quad \lambda \rho \leq c_{\rho 2}, \quad \xi_{e} \geq \xi_{e 2}
$$

then

$$
\dot{\bar{W}}_{\xi_{\sigma}, \xi_{e}}(\bar{x}, \tau) \leq-\frac{1}{2} \min \{\tilde{\alpha}, \epsilon\}|x|^{2}, \quad \forall \bar{x} \text { s.t. } \quad\left[\begin{array}{ccc}
I & 0 & 0 \\
0 & I & 0 \\
0 & 0 & \bar{C}_{p}
\end{array}\right] x \in \mathcal{F}_{y}, \forall \tau \in[0,2 \rho]
$$

Now let us consider (29) and (31). To satisfy them both, so that the analysis in the two cases above holds, we can select $\xi_{\sigma}=c_{\sigma 2}, \lambda=\frac{c_{\sigma 1}}{c_{\sigma 2}}, \xi_{e} \geq \max \left\{\xi_{1 e}, \xi_{2 e}\right\}$ and $\rho^{*}=\frac{c_{\sigma 2}}{c_{\sigma 1}} \min \left\{c_{\rho 1}, c_{\rho 2}\right\}$. Then for any $\rho \leq \rho^{*}(29)$ and (31) hold and the proof of the claim holds too with $\gamma=\frac{1}{2} \min \{\tilde{\alpha}, \epsilon\} \min \left\{1, \lambda_{\min }\left(T_{\zeta}^{T} T_{\zeta}\right)\right\}$.

Proof of Proposition 1 Let us consider item 1. First note that due to the cascaded structure of the system, if $e(0,0)=0$, then $e(t, j)=0$ for all $(t, j) \in \operatorname{dom}(\xi)$. Therefore the observer dynamics does not affect the system and it is sufficient to show that there exists $\rho^{*}>0$ such that all the solutions to the state-feedback hybrid closed loop without dwell-time logic corresponding to [25, Proposition 1] automatically satisfy a dwell time of at least $\rho^{*}$, as long as $x_{p}(0,0) \neq 0$, so that the dwell-time condition does not prevent any jump of the original state-feedback scheme. 
To prove this property, note that $x_{p}(0,0) \neq 0$ implies $x_{p}(t, j) \neq 0$ for all $(t, j) \in \operatorname{dom}(\xi)$, indeed during flows $x_{p}$ asymptotically converges to zero and during jumps it remains unchanged. Since $x_{p}$ is never zero, whenever a jump occurs, so that $\left(x_{p}, x_{c}\right) \in \mathcal{J}$, then $\left(x_{p}^{+}, x_{c}^{+}\right)=\left(x_{p}, K_{p} x_{p}\right)$ belongs to the interior of $\mathcal{F}$ (due to the fact that $\tilde{\alpha} \leq \alpha$ and by the strict inequality in (5)), therefore from continuity it necessarily flows for some time $t_{f}\left(x_{p}\right)$ which depends on the plant state $x_{p}$ at the jump time. Since the dynamics is homogeneous and the flow and jump sets are symmetric cones, then each response can be written as a scaled version of the response starting from the initial condition with unit norm $\frac{x_{p}}{\left|x_{p}\right|}$. Due to this fact, we can compute

$$
\min _{x_{p} \neq 0} t_{f}\left(x_{p}\right)=\min _{x_{p}:\left|x_{p}\right|=1} t_{f}\left(x_{p}\right)=t_{f m}
$$

where we have $t_{f m}>0$ because the minimum is carried out over a compact set and $t_{f}\left(x_{p}\right)>0$ for all $x_{p} \neq 0$. Finally, it is sufficient to pick $\rho^{*} \leq t_{f m}$ to obtain the result at item 1 .

Consider now item 2 and note that, from item 1, any solution to the state feedback hybrid closed loop of [25] is a solution to $(13),(14)$ (resp. (8), (9)) starting from $e(0,0)=0$ and $\tau(0,0) \geq \rho$. Therefore we prove this item by only focusing on two solutions $\xi_{\circ}$ and $\xi_{1}$ to $(13)$, (14) (resp. (8), (9)), where $\xi_{\circ}(0,0)=\left(x_{p 0}, x_{c 0}, 0, \tau_{0}\right)$ and $\xi_{1}(0,0)=\left(x_{p 0}, x_{c 0}, e_{0}, \tau_{0}\right)$. To this aim, we will first establish the result for the case $\left|\left(x_{p 0}, x_{c 0}\right)\right|=1$ and then apply homogeneity to extend it to the whole space.

First note that from global exponential stability of (13), (14) (resp. (8), (9)), uniform convergence implies that for each $\epsilon>0, \exists\left(T_{\epsilon}, J_{\epsilon}\right) \in \mathbb{R}_{\geq 0} \times \mathbb{Z}_{\geq 0}$ such that for all solutions $\xi:=(x, \tau):=\left(x_{p}, x_{c}, e, \tau\right)$ to (13), (14) (resp. (8), (9))

$$
\begin{aligned}
\left|\left(x_{p 0}, x_{c 0}\right)\right| & =1 \\
\left|e_{0}\right| & \leq 1
\end{aligned} \Rightarrow|x(t, j)| \leq \frac{\epsilon}{2}, \quad \forall(t, j) \geq\left(T_{\epsilon}, J_{\epsilon}\right),(t, j) \in \operatorname{dom}(\xi)
$$

Moreover, since the hybrid systems (13), (14) (resp. (8), (9)) satisfy the fundamental conditions $(A 0)-(A 4)$ of [17] and are forward complete, given the two solutions $\xi_{\circ}=\left(x_{\circ}, \tau_{\circ}\right), \xi_{1}=\left(x_{1}, \tau_{1}\right)$ starting from the compact set $\left|\left(x_{p 0}, x_{c 0}\right)\right|=1$, $\left|e_{0}\right| \leq 1$ and $\tau_{0} \in[0,2 \rho]$ and given $\epsilon, T_{\epsilon}, J_{\epsilon}$ in (32), from [17, Corollary 4.8], there exists $\delta_{N}>0$ such that $\left|e_{0}\right| \leq \delta_{N}$ implies that $\xi_{\circ}$ and $\xi_{1}$ are $\left(T_{\epsilon}, J_{\epsilon}, \epsilon\right)$-close. Outside the compact hybrid time domain $\left(T_{\epsilon}, J_{\epsilon}\right)$, from (32), we get

$$
\left|x_{\circ}(t, j)-x_{1}(t, j)\right| \leq\left|x_{\circ}(t, j)\right|+\left|x_{1}(t, j)\right| \leq \epsilon, \quad \forall(t, j) \geq\left(T_{\epsilon}, J_{\epsilon}\right)
$$

Therefore, combining the two bounds above, we have

$$
\begin{aligned}
\left|\left(x_{p 0}, x_{c 0}\right)\right| & =1 \\
\left|e_{0}\right| & \leq \delta
\end{aligned} \Rightarrow\left(x_{\circ}, x_{1}\right) \text { are } \epsilon \text {-close }
$$

where $\delta:=\min \left\{1, \delta_{N}\right\}$. The proof is completed by extending the result to the whole space by using the homogeneity of system (13), (14) (resp. (8), (9)). In particular, noticing that the $x$ component of any solution $\xi=(x, \tau)$, starting from $\xi(0,0)=\left(x_{p 0}, x_{c 0}, e_{0}, \tau_{0}\right)$ with $x_{p 0} \neq 0$, can be written as $\xi=\left(\left|\left(x_{p 0}, x_{c 0}\right)\right| x_{N}, \tau\right)$, with $x_{N}:=\frac{x}{\left|\left(x_{p 0}, x_{c 0}\right)\right|}=\left(x_{p N}, x_{c N}, e_{N}\right)$ satisfying $\left|\left(x_{p N}(0,0), x_{c N}(0,0)\right)\right|=\frac{\left|\left(x_{p 0}, x_{c 0}\right)\right|}{\left|\left(x_{p 0}, x_{c 0}\right)\right|}=1$ and $\left|e_{N}(0,0)\right|=\frac{e_{0}}{\mid\left(x_{p 0}, x_{c 0}\right)}$, the bound in (33) implies that for any $\left|e_{0}\right| \leq\left|\left(x_{p 0}, x_{c 0}\right)\right| \delta$, the components $x_{\circ}, x_{1}$ of $\xi_{\circ}$ and $\xi_{1}$ (therefore, also their $\left(x_{p}, x_{c}\right)$-components) are $\epsilon\left|\left(x_{p 0}, x_{c 0}\right)\right|$-close. 


\section{Conclusions}

The design problem of a hybrid stabilizing loop on a linear continuous-time control system has been considered. The obtained closed-loop system has a mixed discrete/continuous dynamics depending on the value of the output of the system. The hybrid controller is an output feedback since its dynamics depend only on the output of the system. Moreover, it is based on the definition of a pre-computed static state feedback law and of a Luenberger observer which are computed separately. It is known from [25] that, the pre-computed state feedback law allows to define a hybrid feedback allowing to improve the performance (as the output overshoot, or a decreasing property of a given Lyapunov-like function). To embed the estimate of the state given by the observer into the hybrid controller that is computed in [25], a temporal regularization has been used as done in [13] for a different control problem. Some simulations highlighted the interest of the results.

This work lets some questions open. In particular the generalization to nonlinear control systems could be interesting and fruitful. This generalization may be possible by noting that the synthesis method of a hybrid controller of [25] is also valid for nonlinear control systems. However some efforts should be done to adapt the temporal regularization, inspired from [13], to the nonlinear control systems case.

\section{References}

[1] W.H.T.M. Aangenent, G. Witvoet, W.P.M.H. Heemels, M.J.G. van de Molengraft, and M. Steinbuch. Performance Analysis of Reset Control Systems. International Journal of Robust and Nonlinear Control, 20(11):1213-1233, 2010.

[2] A. Anta and P. Tabuada. To Sample or not to Sample: Self-triggered Control for Nonlinear Systems. IEEE Transactions on Automatic Control, 55:2030-2042, 2010.

[3] O. Beker, C Hollot, and Y. Chait. Fundamental Properties of Reset Control Systems. Automatica, 40(6):905-915, 2004.

[4] O. Beker, C.V. Hollot, and Y. Chait. Plant with an Integrator: an Example of Reset Control Overcoming Limitations of Linear Feedback. IEEE Transactions on Automatic Control, 46:1797-1799, 2001.

[5] C. Cai, A.R. Teel, and R. Goebel. Smooth Lyapunov Functions for Hybrid Systems, Part II: (Pre)Asymptotically Stable Compact Sets. IEEE Transactions on Automatic Control, 53(3):734-748, 2008.

[6] Q. Chen, Y. Chait, and C.V. Hollot. Analysis of Reset Control Systems Consisting of a FORE and Second Order Loop. J. Dynamic Systems, Measurement and Control, 123:279-283, 2001.

[7] M.C.F. Donkers and W.P.M.H. Heemels. Output-based Event-Triggered Control with Guaranteed $L_{\infty}$-gain and Improved and Decentralized Event-Triggering. IEEE Transactions on Automatic Control, to appear.

[8] M.C.F. Donkers, W.P.M.H. Heemels, N. van de Wouw, and L. Hetel. Stability Analysis of Networked Control Systems Using a Switched Linear Systems Approach. IEEE Transactions on Automatic Control, 56(9):2101-2115, 2011. 
[9] F. Fichera, C. Prieur, S. Tarbouriech, and L. Zaccarian. A Convex Hybrid $\mathcal{H}_{\infty}$ Synthesis with Guaranteed Convergence Rate. In Proceedings of the 2012 Conference on Decision and Control, submitted, 2012.

[10] F. Fichera, C. Prieur, S. Tarbouriech, and L. Zaccarian. Improving the Performance of Linear Systems by Adding a Hybrid Loop: the Output Feedback Case. In Proceedings of the 2012 American Control Conference, Montreal, Canada, 2012.

[11] F. Fichera, C. Prieur, S. Tarbouriech, and L. Zaccarian. On Hybrid State-feedback Loops Based on a Dwell-time Logic. In 4th IFAC Conference on Analysis and Design of Hybrid Systems, Eindoven, The Netherlands, 2012.

[12] F. Forni, S. Galeani, D. Nešić, and L. Zaccarian. Lazy Sensors for the Scheduling of Measurements Samples Transmission in Linear Closed Loops Over Networks. In Proceedings of the 49th IEEE Conference on Decision and Control, pages 6469-6474, Atlanta (GA), USA, December 2010.

[13] F. Forni, S. Galeani, D. Nešić, and L. Zaccarian. Lazy Sensors: Lyapunov-based Scheduling of Samples Transmission Over Networks. Automatica, 2012 submitted.

[14] G. Franklin, J.D. Powell, and A. Emami-Naeini. Feedback Control of Dynamic Systems, 6Ed. Prentice Hall, 2009.

[15] R. Goebel, R.G. Sanfelice, and A.R. Teel. Hybrid Dynamical Systems. IEEE Control Systems Magazine, 29(2):2893, April 2009.

[16] R. Goebel, R.G. Sanfelice, and A.R. Teel. Hybrid Dynamical Systems: Modeling, Stability and Robustness. Princeton University Press, 2012.

[17] R. Goebel and A.R. Teel. Solutions to Hybrid Inclusions via Set and Graphical Convergence With Stability Theory Applications. Automatica, 42(4):573-587, 2006.

[18] R. Goebel and A.R. Teel. Direct Design of Robustly Asymptotically Stabilizing Hybrid Feedback. ESAIM: Control Optim. Calc. Var., 15(1):205-213, 2009.

[19] J.P. Hespanha, D. Liberzon, and A.S. Morse. Hysteresis-based Switching Algorithms for Supervisory. Automatica, $39(2): 263-272,2004$.

[20] M. Mazo Jr., A. Anta, and P. Tabuada. An ISS Self-triggered Implementation of Linear Controllers. Automatica, $46(8): 1310-1314,2010$.

[21] D. Nešić, A.R. Teel, and L. Zaccarian. Stability and Performance of SISO Control Systems with First Order Reset Elements. IEEE Transactions on Automatic Control, page in press, 2011.

[22] D. Nešić, L. Zaccarian, and A.R. Teel. Stability Properties of Reset Systems. In Proceedings of the 16th IFAC World Congress, volume 16, pages 67-72, Prague, Czech Republic, 2005.

[23] D. Nešić, L. Zaccarian, and A.R. Teel. Stability Properties of Reset Systems. Automatica, 44(8):2019-2026, 2008.

[24] C. Prieur and A. Astolfi. Robust Stabilization of Chained Systems via Hybrid Control. IEEE Transactions on Automatic Control, 48(10):1768-1772, 2003. 
[25] C. Prieur, S. Tarbouriech, and L. Zaccarian. Guaranteed Stability for Nonlinear Systems by Means of a Hybrid Loop. In Proceedings of the 8th IFAC Symposium on Nonlinear Control Systems (NOLCOS), pages 72-77, Bologna, Italy, September 2010.

[26] C. Prieur, S. Tarbouriech, and L. Zaccarian. Improving the Performance of Linear Systems by adding a Hybrid Loop. In 18th IFAC World Congress, Milano, Italy, September 2011.

[27] C. Prieur, S. Tarbouriech, and L. Zaccarian. Lyapunov-based hybrid loops for stability and performance of continuous-time control systems. Automatica, 2012, to appear.

[28] R.G. Sanfelice, R. Goebel, and A.R. Teel. Invariance Principles for Hybrid Systems With Connections to Detectability and Asymptotic Stability. IEEE Transactions on Automatic Control, 52(12):2282-2297, 2007.

[29] P. Tabuada. Event-triggered Real-time Scheduling of Stabilizing Control Tasks. IEEE Transactions on Automatic Control, 52(9):1680-1685, 2007.

[30] S. Tarbouriech, T. Loquen, and C. Prieur. Anti-windup Strategy for Reset Control Systems. International Journal of Robust and Nonlinear Control, 21(10):1159-1177, 2011.

[31] A.R. Teel. Observer-based Hybrid Feedback: a Local Separation Principle. In Proceedings of the 2010 American Control Conference, pages 898-903, Baltimore, Maryland, USA, 2010.

[32] A.R. Teel, F. Forni, and L. Zaccarian. Lyapunov-based Sufficient Conditions for Exponential Stability in Hybrid Systems. IEEE Transactions on Automatic Control, 2012, to appear. 